\title{
Membrane compartmentalization by adherens junctions creates a spatial switch for Notch signaling and function
}

\section{Minsuk Kwak}

University of California San Francisco

Kaden Southard

University of California San Francisco

Woon Ryoung Kim

University of California San Francisco

Annie Lin

University of California San Francisco

Nam Hyeong Kim

Sungkyunkwan University

Ramu Gopalappa

Yonsei University

Minji An

Yonsei University

Hyun Jung Lee

University of California San Francisco

Seo Hyun Choi

Yonsei University

Justin Farlow

University of California San Francisco

Anastasios Georgakopoulos

Icahn School of Medicine at Mount Sinai

Nikolaos Robakis

Icahn School of Medicine at Mount Sinai

Min Kang

University of California San Francisco

Matthew Kutys

University of California San Francisco https://orcid.org/0000-0002-0752-649X

Daeha Seo

DGIST

Hyongbum Henry Kim 
Yonsei University College of Medicine https://orcid.org/0000-0002-4693-738X

\section{Yong Ho Kim}

Sungkyunkwan University

\section{Jinwoo Cheon}

Yonsei University

\section{Zev Gartner}

University of California, San Francisco https://orcid.org/0000-0001-7803-1219

Young-wook Jun ( $\square$ young-wook.jun@ucsf.edu )

University of California San Francisco https://orcid.org/0000-0003-3182-5366

\section{Article}

Keywords: Adherens junctions (AJs), spatial switches, membrane microdomains

Posted Date: November 4th, 2021

DOl: https://doi.org/10.21203/rs.3.rs-956506/v1

License: (c) (i) This work is licensed under a Creative Commons Attribution 4.0 International License. Read Full License 


\section{Membrane compartmentalization by adherens junctions creates a spatial switch for Notch signaling and function}

Minsuk Kwak ${ }^{1,2,3,4,5,6,17}$, Kaden M. Southard ${ }^{1,2,17}$, Woon Ryoung Kim Ki,2,3, $^{\text {, Annie Lin }}{ }^{1,2,3}$, Nam Hyeong $\mathrm{Kim}^{1,2,3,6,7}$, Ramu Gopalappa ${ }^{4,8}$, Minji An ${ }^{4,5}$, Hyun Jung Lee ${ }^{1,2,3}$, Seo Hyun Choi ${ }^{4,5}$, Justin Farlow ${ }^{2}$, Anastasios Georgakopoulos ${ }^{9}$, Nikolaos K. Robakis ${ }^{9}$, Min K. Kang ${ }^{10}$, Matthew L. Kutys ${ }^{11}$, Daeha Seo ${ }^{12}$, Hyeong Bum Kim ${ }^{4,5,8,13,14}$, Yong Ho Kim ${ }^{6,7}$, Jinwoo Cheon ${ }^{4,5,15}$, Zev J. Gartner ${ }^{2,16, *}$, Young-wook Jun $^{1,2,3,4,5 *}$

${ }^{1}$ Department of Otolaryngology, University of California, San Francisco, CA, USA

${ }^{2}$ Department of Pharmaceutical Chemistry, University of California, San Francisco, CA, USA

${ }^{3}$ Helen Diller Family Cancer Comprehensive Center (HDFCCC), University of California, San Francisco, CA, USA

${ }^{4}$ Center for Nanomedicine, Institute for Basic Science (IBS), Seoul, Republic of Korea

${ }^{5}$ Graduate Program of Nano Biomedical Engineering (Nano BME), Advanced Science Institute, Yonsei University, Seoul, Republic of Korea

${ }^{6}$ SKKU Advanced Institute of Nanotechnology (SAINT), Sungkyunkwan University, Suwon, Republic of Korea

${ }^{7}$ Department of Biomedical Engineering, Sungkyunkwan University, Suwon, Republic of Korea ${ }^{8}$ Department of Pharmacology, Yonsei University College of Medicine, Seoul, Republic of Korea ${ }^{9}$ Department of Psychiatry and Neuroscience, Icahn School of Medicine at Mount Sinai, New York, USA

${ }^{10}$ Department of Neurology, University of California, San Francisco, CA, USA

${ }^{11}$ Department of Cell and Tissue Biology, University of California, San Francisco, CA, USA

${ }^{12}$ Department of Emerging Materials Science, DGIST, Daegu, Republic of Korea

${ }^{13}$ Brain Korea 21 Plus Project, Yonsei University College of Medicine, Seoul, Republic of Korea

${ }^{14}$ Severance Biomedical Science Institute, Yonsei University College of Medicine, Seoul, Republic of Korea

${ }^{15}$ Department of Chemistry, Yonsei University, Seoul, Republic of Korea

${ }^{16}$ Chan Zuckerberg Biohub, University of California San Francisco, CA, USA

${ }^{17}$ These authors contributed equally.

*Correspondence: young-wook.jun@ucsf.edu or zev.gartner@ucsf.edu 
Adherens junctions (AJs) create spatially and mechanically discrete microdomains at the interfaces of cells ${ }^{1-6}$. Using a mechanogenetic platform that generates artificial AJs with controlled protein localization, clustering, and mechanical loading, we report that AJs also organize proteolytic hotspots for $\gamma$-secretase with a spatially-regulated substrate selectivity that is critical in the processing of Notch and other transmembrane proteins. Membrane microdomains outside of AJs exclusively organize Notch ligand-receptor engagement (LRE-

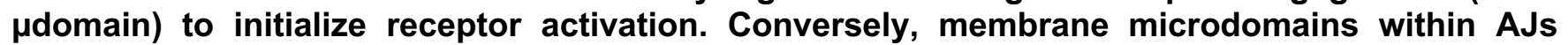

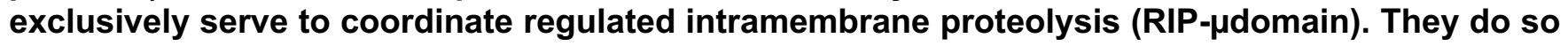
by concentrating $\gamma$-secretase and primed receptors while excluding full-length Notch. AJs induce these functionally distinct microdomains by means of cholesterol-dependent $\gamma$-secretase recruitment and size-dependent protein segregation. By excluding full-length Notch from RIP$\mu$ domains, AJs prevents inappropriate enzyme-substrate interactions and suppresses spurious Notch activation. Ligand-induced ectodomain shedding eliminates size-dependent segregation, releasing Notch to translocate into AJs for processing by $\gamma$-secretase. This mechanism directs radial differentiative expansion of ventricular zone-neural progenitor cells in vivo and more broadly regulates the proteolysis of large cell-surface receptors like amyloid precursor protein. These findings suggest an unprecedented role of AJs in creating size-selective proteolytic hotspots that choreograph $\gamma$-secretase processing of multiple transmembrane proteins regulating development, homeostasis, and disease. 
Juxtacrine signaling occurs between cells that are in direct physical contact and orchestrates a wide range of cellular processes involved in development, physiology, and disease across multiple multicellular organisms ${ }^{4,7}$. For example, Notch is a prototypical juxtacrine signaling pathway that frequently serves to amplify cellular differences, tipping the scales during cell fate determination and enabling pattern formation during development. Similarly, juxtacrine neural adhesion and immune receptors are also critical in establishment and maintenance of neurological ${ }^{8-11}$ and immunological synapses $^{12,13}$, regulating the neurological and immunological functions, respectively.

Unlike soluble ligand-receptor interactions that globally stimulate cells by diffusion, juxtacrine ligand-receptor pairs localize cell signaling activities to a signal-exchange interface. As two juxtaposed membranes are coupled chemically, spatially, and mechanically, the signal-exchange interface undergo drastic reorganization that constrains the arrangement and activity of both protein and lipid components at the interface ${ }^{14-16}$. Cells can exploit these interfacial constraints to form specialized membrane compartments that regulate receptor activation, as exemplified by the kinetic segregation and liquid-liquid phase separation (LLPS) models of immune receptor signaling ${ }^{17-23}$. However, many cellular interfaces beyond the immunological synapse can also create these constraints, but the structural and functional consequence of such constraints on other signaling pathways have not been explored extensively.

Here, we report specialized interfacial membrane compartments organized by adherens junctions (AJs) that create two physically and biochemically distinct microenvironments for the sequential molecular processing of Notch: One serves as a microdomain for ligand-receptor engagement (here and

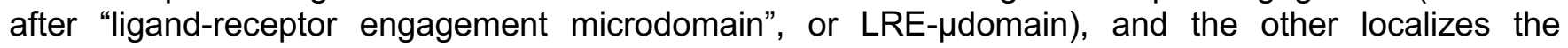
proteolytic activity of $\gamma$-secretase while effectively selecting only primed receptors for cleavage via sizedependent exclusion (here and after "receptor intramembrane proteolysis microdomain", or RIP$\mu$ domain). By employing mechanogenetics, spatial mutation, and CRISPR-Cas9 knockout approaches, we show that this membrane micro-compartmentalization choreographs the sequential activation of Notch both in vitro and in vivo. We also demonstrate the generality of this model to other large cell surface proteins by examining the role of AJs in amyloid precursor protein (APP) proteolysis promoting pathogenic amyloid beta $(A \beta)$ production.

\section{Key steps in cell surface activation of Notch occur in two mutually exclusive and functionally distinct membrane microdomains.}

Notch is a highly conserved mediator of contact-dependent cell-cell communication, which orchestrates diverse functions in metazoans ${ }^{24-27}$. Tight control of Notch signal activation is essential for many developmental processes ${ }^{28,29}$, while dysregulation of Notch activation can cause severe disease including developmental, neurological, and immunological disorders and cancer ${ }^{25,30-33}$. Accordingly, to enable precise signal regulation, cell-surface receptor activation occurs through multiple steps that are independently gated by sequential events including (i) ligand-receptor engagement, (ii) mechanical unfolding of the negative regulatory region (NRR) and proteolytic extracellular domain shedding (S2 cleavage), and (iii) regulated intramembrane proteolysis (S3 cleavage) finally releasing Notch intercellular

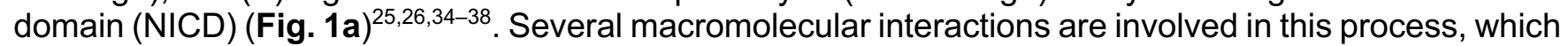
include Notch ligands, metalloprotease (e.g., ADAM 10/17), and $\gamma$-secretase.

To interrogate the spatial organization of these molecules at cellular interface, we generated a series of U2OS cells expressing recombinant Notch1 and/or DIl1 proteins fused with self-labeling tags (SNAP or Halo-tags) and/or fluorescent proteins (GFP or mCherry) at the N- or C-termini, respectively (Supplementary Table 1). We formed Notch signaling interfaces by culturing cells co-expressing both SNAP-Notch and Halo-DII1 and labeled cells with SNAP- and Halo-specific fluorescence dyes. We prevented Notch proteolysis and activation by treating cells with TAPI2, an ADAM10/17 inhibitor, to explicitly visualize full-length Notch. The endogenous Notch-processing enzymes (i.e., ADAM 10/17, and $\gamma$-secretase) were immunostained and then imaged by confocal microscopy. Enriched fluorescence signals for SNAP-Notch and Halo-DII1 were seen at the cellular interface, indicating accumulation of the engaged ligand-receptor pairs at the interface (i.e., LRE- $\mu$ domain) (Fig. 1a,b). Conversely, we also observed regions within the interfacial membrane that were depleted of both fluorescence signals, suggesting that certain interfacial microdomains exclude Notch ligands and receptors (Fig. 1a,b). 
Surprisingly, the punctuated exclusion patterns matched cell-surface $\gamma$-secretase distribution, visualized with an anti-presenilin-1 (anti-PS1) antibody (Fig. 1a,b). This result indicates that Notch ligand-receptors and $\gamma$-secretase were compartmentalized to two different membrane microdomains at the signaling interface and hence interactions between Notch receptors and $\gamma$-secretase were prevented. Calculated Manders' overlap coefficients (MOC) over $\gamma$-secretase were $0.09 \pm 0.083$ and $0.1 \pm 0.08$ for Notch and DII1, respectively, confirming the exclusion of Notch and DII1 from the microdomains enriched with $\gamma$ secretase, the enzyme responsible for the final proteolytic step necessary for Notch activation (Fig. 1c).

Considering the requirement of Notch proteolysis by $\gamma$-secretase for its activation, we reasoned that cell-surface molecular processing steps for Notch activation (ligand-receptor engagement vs. S3 receptor intramembrane proteolysis) may occur within distinct membrane regions. To test this notion, we investigated how each cleavage step during activation alters the spatial distribution of Notch. To induce robust cell surface activation of Notch, we plated U2OS cells expressing SNAP-Notch-mCherry on a DII4coated substrate (Fig. 1d). To promote accumulation of specific Notch activation intermediates following ADAM 10/17 and $\gamma$-secretase cleavage, we treated cells with TAPI2 (S2 cleavage inhibition) or DAPT ( $\gamma$ secretase inhibitor, S3 cleavage inhibition), respectively (Fig. 1d). With TAPI2, we observed Notch exclusion from microdomains enriched with $\gamma$-secretase (Extended Data Fig. 1a). In contrast, when TAPI2 was removed to activate S2 cleavage and DAPT was added to inhibit S3 cleavage, we observed strong enrichment of mCherry signal within the $\gamma$-secretase-containing microdomain (Fig. 1e), indicating that Notch with extracellular domain truncation (NEXT), the product of S2-cleavage, translocated to and was then concentrated within the microdomains. When $\gamma$-secretase activity was rescued by washing out DAPT, the mCherry signal previously enriched at the $\gamma$-secretase-containing microdomain disappeared (Extended Data Fig. 1a), presumably corresponding to release of Notch intracellular domain (NICD) resulting from S3-proteolysis of accumulated Notch within the $\gamma$-secretase-containing microdomain (i.e.,

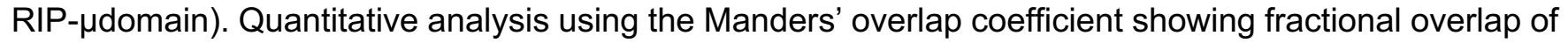
mCherry signal with $\gamma$-secretase also confirmed translocation of Notch from LRE- to RIP- $\mu$ domains, and then NICD release intracellularly (Fig. 1f). These results support the notion that these two membrane microdomains serve distinct and necessary functions in the activation of Notch receptors. They also raise the possibility that movement of Notch between these domains serves as a spatial switch that regulates the interaction between Notch intermediates and $\gamma$-secretase, thereby choreographing sequential steps in Notch proteolysis. According to this model, $\gamma$-secretase cannot process the full-length Notch before S2 cleavage because enzyme and substrate are concentrated in distinct regions of the cell surface. Following S2 cleavage, translocation of NEXT into the RIP- $\mu$ domain facilitates a productive Notch- $\gamma$ secretase interaction, S3 cleavage, and then downstream signaling.

\section{Adherens junctions organize Notch signaling molecules at cellular interface}

To gain insight into molecular features responsible for organizing these distinct membrane microdomains we imaged $\gamma$-secretase distribution across entire cell membranes. Interestingly, we observed many microdomains with strong $\gamma$-secretase signals at the cell-cell interfacial membranes, but detected negligible $\gamma$-secretase signals within cell membranes without contact, suggesting that the RIP- $\mu$ domains were formed at cellular interface exclusively (Extended Data Fig. 1b). Since the cell-cell interface is established and maintained by adherens junctions (AJs) in many tissues ${ }^{1,2,5,39}$, we reasoned that AJs

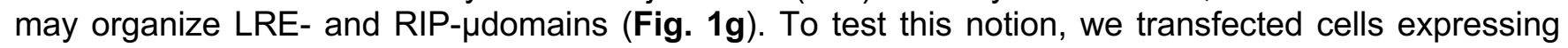
SNAP-Notch and/or Halo-DII1 proteins with plasmids encoding epithelial cadherin fused with GFP (Ecad-

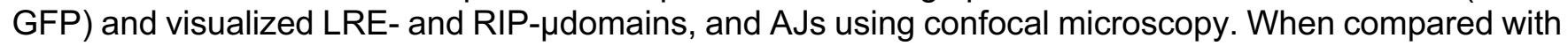
AJs, RIP- domains containing $\gamma$-secretase showed nearly identical spatial distribution (Fig. 1h;

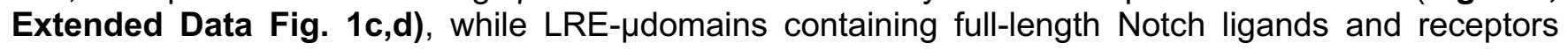
exhibited inverse distribution (Fig. 1h and Extended Fig. 1e and 1f). ADAM 10/17 exhibited no preferential distribution relative to AJs (Extended Fig. 1g-i). Quantitative analysis using the Manders' overlap coefficient to calculate fractional overlap with cadherin also confirmed the positive correlation with $\gamma$-secretase $(0.85 \pm 0.21)$ vs. E-cadherin and the inverse correlation with Notch $(0.24 \pm 0.19)$ and Dll1 $(0.26 \pm 0.18)$ (Fig. 1i). We also confirmed that Notch exclusion from AJs was observed in multiple contexts, including endogenous vs. recombinant expression of Notch (Extended Data Fig. 1j), different 
cadherin types, cell types, and cell polarization states (Extended Data Fig. 1k-m), supporting the generality of AJ-mediated microdomain formation. Moreover, we observed Notch translocation into AJs after S2-cleavage, consistent with Notch relocalization from LRE- to RIP- $\mu$ domains (Extended Data Fig. 2a-e; Supplementary Note 1). These observations suggest two mechanisms by which AJs might define the compartmentalized microdomains: first, AJs recruit $\gamma$-secretase that forms RIP- $\mu$ domain; second, AJs

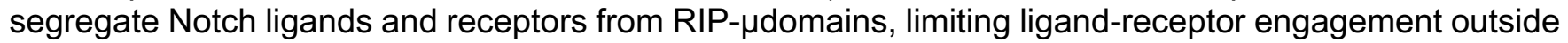

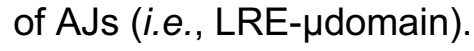

\section{AJs form RIP- $\mu$ domains by recruiting $\gamma$-secretase through cholesterol-rich lipid assemblies}

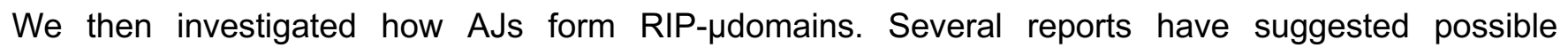
engagement of AJs with spatially discrete lipid membrane phases ${ }^{5,40-42}$. Similarly, $\gamma$-secretase proteolytic activity is closely linked to detergent-resistant membranes ${ }^{43-50}$. Both of these membrane features preferentially associate with membrane proteins such as Flotillin-1 (Flot1) ${ }^{5,48-51}$. We therefore visualized localization of Flot1 across the cell membrane. Strong Flot1 and $\gamma$-secretase fluorescence signal was seen at AJs (Fig. 2a,b and Extended Data Fig. 2f,g). These observations support the notion that both AJs and $\gamma$-secretase are associated with common and long-lived lipid membrane phases enriched with Flot1, otherwise known to be short-lived and transient when alone ${ }^{5}$. Clustering of E-cadherin triggers rapid F-actin polymerization at the cytoplasmic leaflet of the plasma membrane. Given the established interaction between F-actin and membrane constituents like phosphatidylserine that nucleate and stabilize Flot1-containing lipid microdomains, we reasoned that AJ components may anchor phosphatidylserine leading to formation of the membrane microdomains (Raghupathy et al., 2015; Yap et al., 2015). To test this notion, we performed a coarse-grained molecular dynamic (MD) simulation of a lipid membrane comprising of 1,2-dilinoleoyl-sn-glycero-3-phosphocholine (DIPC; outer leaflet), 1,2dipalmitoyl-sn-glycero-3-phosphocholine (DPPC; outer leaflet), N-palmitoyl-O-phosphocholineserine (PPCS; outer leaflet), 1,2-dipalmitoyl-sn-glycero-3-phosphoserine (DPPS; inner leaflet), and cholesterol (Chol; inner and outer leaflet, a key component of the discrete lipid membrane phases). We immobilized a portion (30\%, red colored in Fig. 2c) of DPPS in the inner leaflet to reflect its interaction with AJ components (e.g., F-actin) and compared the results to another simulation set without DPPS immobilization. While both simulations showed lipid segregation within both the inner and outer leaflets, DPPS immobilization resulted in a microdomain having a strong transbilayer coupling (Fig. 2c) ${ }^{52}$. We also observed a significant decrease in lipid diffusion, indicating the microdomain stabilization (Fig. 2d,e).

To further confirm the role of discrete lipid membrane phases in recruiting $\gamma$-secretase to AJs, we tested whether cholesterol-depletion disrupts $\gamma$-secretase localization within AJs. Because cholesterol depletion also destabilizes native AJs ${ }^{50}$, we instead employed a mechanogenetic approach to maintain E-cadherin clustering while simultaneously depleting cholesterol ${ }^{38,53,54}$ (Fig. 2f,g). We treated cells with chloroalkane-functionalized magnetofluorescent nanoparticles (MFNs) to selectively label Halo-tagged E-cadherin in the presence of methyl- $\beta$-cyclodextrin $(M \beta C D)$ that depletes cholesterol in the cell membrane. We then triggered E-cadherin clustering by applying a focused magnetic field to a certain subcellular location, generating artificial AJs (Fig. 2f,g). Similar to native AJs, vivid Flot1 and PS1 signals were seen at the artificial AJ without M $\beta C D$ treatment (Fig. 2h; Extended Data Fig. 2h,i), indicating that artificial AJs recapitulate the functional and signaling roles of native cell-cell AJs including $\gamma$-secretase recruitment. In contrast, when cells were treated with MBCD, neither Flot1 nor PS1 signals were detected at the artificial AJ (Fig. 2h), suggesting that $\gamma$-secretase recruitment into AJs requires cholesterol-rich lipid assemblies. From these observations, we concluded that AJs form RIP- $\mu$ domains by recruiting and stabilizing $\mathrm{Y}$-secretase through a common spatially discrete lipid assembly.

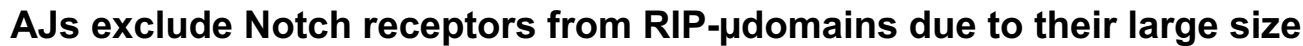

To interrogate how AJ drives receptor exclusion, we generated artificial AJs using mechanogenetics, which allows quantitative control over the location and mechanical loading of targeted receptors while monitoring spatial distribution of Notch signaling components (Fig. 3a) ${ }^{38,53,54}$. Importantly, artificial AJs are free of membrane juxtaposition. Therefore, the influence of membrane juxtaposition on protein 
localization can be investigated by comparing receptor distributions in native AJs to artificial AJs. To image full-length Notch explicitly, we prevented proteolytic processing by treating cells with TAPI2. Surprisingly, we observed intense Notch localization at artificial AJ (Fig. 3b and Extended Data Fig. 3ac), which contrasted starkly with the exclusion of Notch from native AJs (Fig. 1g-i). The extraordinarily large size of the Notch extracellular domain (NECD) ${ }^{55}$ suggested a potential explanation for these contradicting observations. Specifically, the narrow intermembrane cleft created by native AJs $(20 \mathrm{~nm})^{1,3}$. In contrast, artificial AJs generated by MFNs lack a narrow intermembrane cleft, thus permitting access of Notch to the concentrated $\gamma$-secretase and other components of the AJs ${ }^{56}$. These observations fit a model wherein the size-based physical constraint induced by AJ formation at cell-cell interface

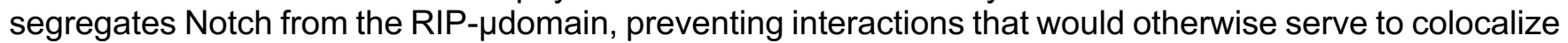
and concentrate the enzyme-substrate pair ${ }^{15}$.

To test a size-dependent model for Notch exclusion from AJ-induced RIP $\mu$ domains, we generated a series of U2OS cell lines stably expressing Notch variants with different truncation lengths: a partial deletion of the EGF repeats (N $\Delta \mathrm{EGF}_{1-25}$, approximate height: $48 \mathrm{~nm}$ ), complete deletion of the EGF repeats but retention of negative regulatory region (N $\triangle E G F$, approximate height: $10 \mathrm{~nm}$ ), and a complete removal of NECD (NEXT, approximate height: $4 \mathrm{~nm}$ ) (Fig. 3c and Extended Data Fig. 3d). We fused these Notch variants with SNAP- and mCherry-tags at their $\mathrm{N}$ - and C-termini, to differentially image the extracellular and intracellular domains. All cells were treated with TAPI2 and DAPT to prevent any potential proteolysis of the variants. To quantify the spatial distribution of each Notch variant relative to the AJ, we measured the average mCherry fluorescence signal inside (liN) and outside (lout) of the AJ and estimated an enrichment ratio ( $\mathrm{I}_{\mathrm{IN}} / \mathrm{l}_{\mathrm{OUT}}$, See METHODS for the details) where a value of 1 indicates homogenous distribution (Extended Data Fig. 3e,f). Consistent with predictions based on sizedependent protein segregation, $\mathrm{N} \triangle \mathrm{EGF}_{1-25}$, the Notch variant with an ECD taller than the height of the intermembrane AJ cleft, was excluded from AJs ( $\left.\mathrm{I}_{\mathrm{IN}} / \mathrm{l}_{\mathrm{OUT}}=0.57\right)$ (Fig. 3d,e). NEXT with an ECD smaller than the junctional height was enriched at $A J s\left(I_{\text {IN }} / l_{\text {OUT }}=2.39\right)($ Fig. 3d,e). Interestingly, we observed a mixed binary localization pattern of $\mathrm{N} \triangle \mathrm{EGF}$ (intermediate height) relative to AJs, with a mean $\mathrm{I}_{\mathrm{IN}} / \mathrm{l}_{\text {OUT }}$ value of 1.32 (Fig. 3d,e). Some AJs displayed NAEGF enrichment (Fig. 3d(bottom left), e(right)), consistent with the size-based prediction. Meanwhile, other AJs excluded N $\triangle E G F$ (Fig. 3d(bottom right), and $\mathbf{e}($ right)). These results suggest a role for the size-dependent protein segregation as a spatial switch that regulates the distribution of Notch intermediates relative to RIP- $\mu$ domain, thereby choreographing the sequential steps in Notch proteolysis. Initially, the large size of NECD presents a physical constraint preventing entry of Notch to the narrow space between membranes in the AJ cleft (i.e., RIP- $\mu$ domain),

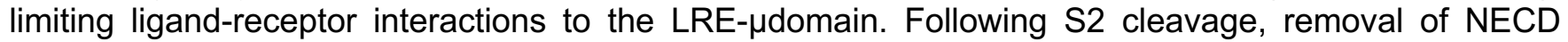
relieves the physical constraint, allowing Notch to enter into the AJ cleft. This facilitates a productive Notch- $\gamma$-secretase interaction, S3 cleavage, within the RIP- $\mu$ domain and then downstream signaling.

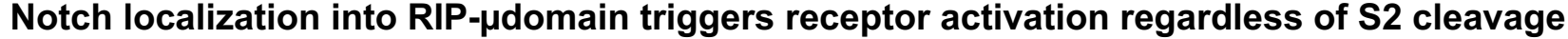

We next interrogated the functional consequences of the differential localization of Notch variants with

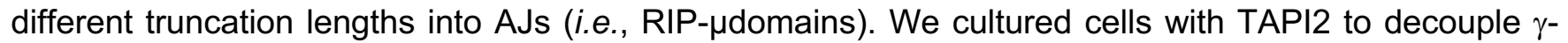
secretase processing from S2 cleavage, and measured cleaved NICD levels by immunoblotting with Notch antibodies that detect $\mathrm{N}$-terminal $\mathrm{V} 1744$. Cells expressing $\mathrm{N}^{\mathrm{FL}}$ or $\mathrm{N} \Delta E \mathrm{GF}_{1-25}$ resulted in no or minimal NICD, respectively (Fig. 3f,g and Extended Data Fig. 3d), whereas cells expressing N $\Delta E G F$ produced a significant amount of NICD (Fig. 3f,g). We validated these findings using time-lapse confocal microscopic tracing of the intracellular mCherry-tag signal within AJs after removing DAPT (Extended Data Fig. 3g-I, Supplementary Note 2). Importantly, we observed a dramatic decrease in the mCherry signal within AJs but not outside AJs, confirming that $\gamma$-secretase activity was localized within the RIP$\mu$ domains (Extended Data Fig. 3i). Cells expressing NEXT exhibited the highest NICD production, about a four-fold increase compared with that of N $\triangle$ EGF (Fig. 3f,g and Extended Data Fig. 3d). The observed NICD production was proportional to the enrichment ratio ( $\left.\mathrm{I}_{\mathrm{IN}} / \mathrm{l}_{\mathrm{OUT}}\right)$ of the Notch variants at AJs, suggesting the essential role of size-dependent protein segregation as a spatial switch to direct Notch activation. The substantial NICD production from the cells expressing N $\triangle E G F$ indicates that, when localized together, $\gamma$-secretase can process Notch, bypassing S2 cleavage. Size-dependent but ligandindependent activation of Notch receptors with an intact S2 site was observed previously in Notch variants 
and synNotch constructs ${ }^{57-61}$, but the mechanism of this activation has been unclear. Our observations support the notion that colocalization of these Notch variants with $\gamma$-secretase is sufficient to trigger S3 proteolysis and signaling.

\section{Spatial mutations alter Notch signaling}

Prevailing models of Notch proteolysis by $\gamma$-secretase are based on the notion that S2-cleavage of Notch serves to potentiate the cleavage by modifying the molecular interface at the enzyme-substrate pair ${ }^{57,61,62}$. For example, it has been suggested that $\gamma$-secretase selectively recognizes S2-cleaved Notch (i.e., NEXT) through hydrogen bonding between a glutamate residue in nicastrin and the new N-terminus of NEXT ${ }^{62}$. Another model proposes that S2 cleavage serves to reduce steric repulsion between nicastrin and NECD, strengthening their interaction ${ }^{61}$. However, another key feature of S2 cleavage is that it generates a smaller molecular intermediate that can uniquely access AJs, thereby colocalizing Notch with $\gamma$-secretase and significantly increasing its concentration near the enzyme active site. This suggests a third model, wherein $\gamma$-secretase activity on full length Notch and its intermediates is blocked by maintaining concentrations of Notch below the $\mathrm{K}_{\mathrm{M}}$ for $\gamma$-secretase due to their compartmentalization within LRE- and

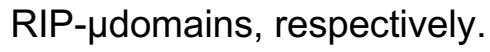

To explicitly test the consequences of membrane compartmentalization on $\gamma$-secretase processing and signaling, we designed three experiments that induce spatial mutations of Notch. First, we employed a DNA-mediated crosslinking strategy to enhance N $\Delta \mathrm{EGF}$ - a Notch variant that exhibited a binary localization relative to AJs and relatively low Notch activation - enrichment at the RIP- $\mu$ domain (i.e., AJ) ${ }^{63}$. We generated cells co-expressing SNAP-N $\triangle E$ EF-mCherry and Halo-Ecad-GFP and promoted colocalization of these two molecules by treating the cells with complementary benzylguanine (BG)- and chloroalkane-modified oligonucleotides in the presence of TAPI2 and DAPT (Fig. 4a). NotchE-cadherin heterodimers were formed efficiently as evidenced by the appearance of a higher molecular weight band corresponding to the DNA-linked complex on western blots (Extended Data Fig. 4a,b). Compared to untreated cells $\left(\mathrm{I}_{\mathrm{IN}} / \mathrm{l}_{\mathrm{OUT}}=1.32 \pm 1.06\right)$, we observed further enrichment of N $\Delta \mathrm{EGF}$ at $A J \mathrm{~J}$ in the presence of DNA crosslinking $\left(\mathrm{I}_{\mathrm{IN}} / \mathrm{l}_{\mathrm{OUT}}=1.89 \pm 0.91\right)(\mathbf{F i g} . \mathbf{4 b})$. We then maintained cells in TAPI2 but removed DAPT to allow S3 cleavage. We observed a decrease in mCherry signal at AJs after DAPT removal, indicating efficient S3 cleavage without S2 cleavage (Extended Data Fig. 4c-e). Accordingly, in western blots, we observed increased V1744-terminated NICD levels from the cells treated with DNA crosslinkers, compared with the untreated control (Fig. 4c). These results suggest that the new molecular interfaces produced by mechanical activation leading to $S 2$ cleavage are not necessary when $\gamma$-secretase is concentrated with its substrate. Considering that DNA crosslinking (molecular weight $=21.4 \mathrm{kD}$ ) increases the ECD size of N $\triangle E G F$, the observed increase in NICD production cannot be explained by the nicastrin-induced steric repulsion model. Rather, this result favors a model wherein the increased

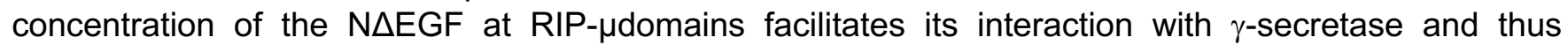
promotes S3 cleavage.

To further test the importance of size-dependent spatial segregation, we induced spatial mutation of NEXT (i.e., the Notch variant that showed the strongest enrichment at the AJ and activation) by chemically conjugating it with macromolecules of increasing hydrodynamic size: polyethylene glycol with an average molecular weight of $3.4 \mathrm{kD}$ (PEG3.4k, $2.5 \mathrm{~nm}$ ), branched PEG20k (bPEG20k, $4.0 \mathrm{~nm}$ ), linear PEG20k ( $\ell$ PEG20k, $8.0 \mathrm{~nm}$ ), DNA-streptavidin conjugates (DNA-stv, $9.5 \mathrm{~nm}$ ), and human immunoglobulin G (hlgG, 12 nm) (Fig. 4d; Extended Data Fig. 4f). Specifically, we conjugated BG-modified polymers and proteins to the extracellular SNAP tag $(4.0 \mathrm{~nm})$ of the variant via BG-SNAP chemistry (Fig. 4d). Grafting of these macromolecular pendants onto NEXT increases the size of the Notch construct but does not modify the N-terminal amine for hydrogen bonding with nicastrin. In the presence of DAPT, we observed a size-dependent distribution of NEXT at the AJ, where the larger pendants resulted in a greater decrease in mCherry signal at the AJ. With pendants smaller than $5 \mathrm{~nm}$ (i.e., PEG3.4k and $b$ PEG20k), NEXT remained enriched at the $A J$ with $I_{\text {IN }} / l_{\text {Out }}$ of 2.21 and 2.01 , respectively (Fig. 4d,e). When $\ell P E G 20 k$, DNA-stv, or hlgG were added, we observed a binary localization pattern of NEXT (i.e., enriched at or excluded from the AJs) with mean $\mathrm{I}_{\mathrm{IN}} / \mathrm{l}_{\mathrm{OUt}}$ values of 1.06, 0.82 , or 0.98 , respectively (Fig. $\left.4 \mathbf{d}, \mathbf{e}\right)$. These observations were similar to the mixed spatial behavior of N $\triangle E G F$ having a comparable ECD size, where 
only a subset of AJs allowed Notch colocalization. We then examined the signaling consequences for each spatial mutation of NEXT. Following S3 cleavage, NICD traffics to the nucleus, allowing us to measure nuclear the mCherry signal as a proxy for Notch pathway activation. The PEG3.4k or $b$ PEG20k addition did not significantly alter nuclear mCherry signal of NEXT, compared with cells with no pendant addition (Fig. 4f,g and Extended Data Fig. 4g). Conjugation of $\ell$ PEG20k and DNA-stv resulted in a substantial decrease in nuclear mCherry signal to 0.39 and 0.37 fractional intensity, respectively (Fig. 4f,g and Extended Data Fig. 4g). hlgG addition suppressed nuclear mCherry signal further to 0.27 (Fig. $\mathbf{4 f , g}$ and Fig. $\mathbf{4 g}$ ). We summarized the NICD production for all Notch variants as a function of the Notch enrichment factor, $\mathrm{I}_{\mathrm{IN}} / \mathrm{l}_{\mathrm{OUt}}$, in Fig. 4h, clearly visualizing the spatial dependence of S3 cleavage.

Finally, we investigated whether $\gamma$-secretase can process full-length Notch when specifically directed to AJs by mechanogenetics. In this case, the biophysical constraints to Notch localization caused by membrane juxtaposition are eliminated and hence no exclusion of Notch receptors from RIP- $\mu$ domains is expected (Fig. 3a). We generated artificial AJs on the cells expressing SNAP-N ${ }^{\mathrm{FL}}$-mCherry using mechanogenetics, in the presence of TAPI2 (to prevent S2 cleavage) but without DAPT (to allow $\gamma$ secretase activity). Contrary to the experiment with DAPT (Fig. 3b), we observed no enrichment of mCherry signal at the artificial AJ, presumably due to NICD release (Fig. 4i,j and Extended Data Fig. $\mathbf{4 h}, \mathbf{i})$. To confirm that the loss of mCherry signal corresponded to bona fide signaling from Notch, we employed a UAS-Gal4 reporter system that detects Notch activation with the nuclear mCherry fluorescence ${ }^{37,38,64,65}$. To a cell recombinantly expressing SNAP-Notch-Gal4 (SNAP-N ${ }^{F L}$-Gal4) and HaloEcad-GFP, we again generated artificial AJs via mechanogenetics and measured the nuclear mCherry fluorescence every two hours. Note that no source of S2 cleavage (e.g., no ligand-immobilized substrate) was added. We observed strong nuclear mCherry signal from the cells with artificial AJs, but no signal from neighboring cells (Fig. 4k,I and Extended Data Fig. 4j). Together, these results suggest that the AJ-mediated membrane microcompartmentalization at cellular interface serves as a critical substrateselection mechanism for $\gamma$-secretase.

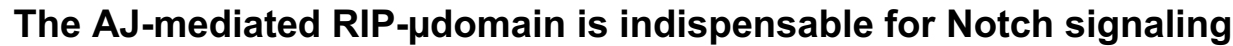

Given the significant role of AJs creating LRE- and RIP- $\mu$ domains, we next interrogated Notch signal activation in cells lacking AJs. In a first experimental approach we minimized physical contact between cells, and hence AJ formation, by sparsely plating UAS-Gal4 reporter cells expressing SNAP-N ${ }^{\mathrm{FL}}-\mathrm{Gal} 4$ on a Dll4-coated substrate - effectively decoupling cell-cell contact from Notch-DII4 interactions by allowing ligand presentation from the glass substrate rather than neighboring cells. After 16 hours from cell seeding, we measured the mCherry fluorescence in cells having no prior contact with other cells. For comparison, we also analyzed the mCherry signal in cells with robust AJs within high-density culture. While cells with physical contacts with adjacent cells exhibited a robust increase in nuclear mCherry fluorescence signal, those without cell-cell contact elicited no increase in signal (Fig. 5a-c and Supplementary Video S1). Reestablishing AJs by plating cells on a substrate coated with Ecad-Fc and DIl4-Fc rescued the Notch signaling of the solitary cells (Fig. 5a,d and Supplementary Video S2). We further confirmed AJ-dependent Notch activation in cells cultured with heterogeneous densities across a DIl4-coated substrate (Fig. 5b). These results support a model wherein AJs (or some other means of enriching $\gamma$-secretase to form RIP- $\mu$ domain) are required for Notch processing at the cell surface and downstream signaling. Critically, E-cadherin seems to function in this capacity in a manner that is independent of its role in mediating cell-cell contact. In a second experimental approach, we knocked out the gene encoding $\mathrm{E}$ - and $\mathrm{N}$-cadherin $(\mathrm{CDH} 1 / 2)$ in the reporter cell line via CRISPR-Cas9 (Extended Data Fig. 5a-d), then plated the cells at high density on Dll4-Fc coated plates. Strikingly, E-cadherin knockout (Ecad-KO) resulted in abrogation of Notch activation even with robust cell-cell contact (Fig. 5e,f and Extended Data Fig. 5e-g). Reintroduction of plasmids encoding E-cadherin or N-cadherin into Ecad$\mathrm{KO}$ cells recovered Notch activation with substantial nuclear mCherry signal (Fig. 5e,f and Extended Data Fig. 5e-g). Single cell analysis of the nuclear fluorescence signal exhibited a clear positive correlation with E-cadherin expression in the respective cells, confirming AJ-dependent Notch signaling (Fig. 5g). 


\section{AJ-mediated membrane compartmentalization regulates neuronal progenitor cell differentiation in vivo}

Notch signaling is essential for the maintenance of stemness, self-renewal, and differentiation of neural progenitor cells (NPCs) ${ }^{66,67}$. In the mammalian cerebral cortex, Notch signaling orchestrates developmental neurogenesis, where it modulates a balance between tangential proliferative (i.e., symmetric division) and radial differentiative (i.e., asymmetric division) expansion of the apical ventricular-zone NPCs (VZ-NPCs) to establish a stratified neuronal organization ${ }^{68}$. Interestingly, only radial expansion of VZ-NPCs accompanies its differentiation, suggesting that Notch signaling in VZ-NPCs may be coupled with cells' spatial cues. Several reports also emphasize the critical role of apical-endfoot AJs in Notch signaling and the decision-making process of VZ-NPC development (i.e., proliferation vs. differentiation) ${ }^{69-71}$.

Given the essential role of the AJ-mediated microdomain formation for Notch signaling in cell line models, we reasoned that apical-endfoot AJs may also organize proteolytic hotspots for Notch activation. To test this hypothesis, we mapped the spatial distribution of Notch and $\gamma$-secretase relative to $\mathrm{N}$ cadherin-based AJs in VZ-NPCs of the developing mouse brain (E13.5) (Fig. 6a-g). Consistent with observations in cell lines, Notch and PS1 exhibited exclusion (MOC $=0.14 \pm 0.05, n=9)$ from and enrichment $(\mathrm{MOC}=0.69 \pm 0.07, \mathrm{n}=9$ ) within AJs, respectively, confirming compartmentalization between LRE- and RIP- $\mu$ domains (Fig. 6c-g). We also captured the spatial distribution of the Notch activation intermediate by intracerebroventricular injection of DAPT into postnatal mice (P3). The immunostaining showed inclusion of Notch signal within AJs, presumably resulting from NEXT accumulation (Extended Data Fig. 6a) as observed in cell lines (Extended Data Fig. 2a,b). These results support the notion that AJs also drive compartmentalized microdomains and serve as a spatial switch regulating Notch signaling in vivo.

To understand the function of the AJs on VZ-NPC development, we disrupted AJs via dominantnegative cadherin expression preventing the RIP- $\mu$ domain formation. We retrovirally transfected a plasmid encoding a dominant-negative form of E-cadherin with the extracellular domain truncation (DNcad) ${ }^{71}$ and a C-terminal GFP tag to VZ-NPCs of developing mice (P3) via intracerebroventricular injection (Fig. 6h). 48 hours after transfection, we analyzed NPC differentiation via TuJ1 immunostaining, a neuronal marker. While mice transfected with a control plasmid $(n=3)$ showed negligible TuJ1 signal, those with DN-cad plasmid transfection $(n=5)$ exhibited robust TuJ1 expression, presumably through downregulation of Notch signaling (Fig. 6i-k and Extended Data Fig. 6b,c). These results support that AJ-mediated membrane microdomain compartmentalization modulates NPC maintenance and differentiation via Notch signaling.

\section{Size-dependent spatial dynamics and proteolysis of amyloid precursor proteins}

To test whether AJs serve as proteolytic hotspots with size dependent substrate selectivity for other large cell surface proteins, we investigated the processing of amyloid precursor protein (APP). APP plays a central role in amyloid beta $(A \beta)$ pathology, which can cause failures in many organs such as brain, heart, kidney, and vasculature ${ }^{72-75}$. Interestingly, APP has a strikingly similar topology and proteolytic cleavage sequence to that of Notch. Like Notch, upon activation, APP is processed by two rounds of proteolysis: first $\alpha$ - or $\beta$-secretase and then $\gamma$-secretase releasing its extracellular and intracellular domains, respectively ${ }^{72,73,75}$. We generated U2OS cells co-expressing APP-GFP and SNAP-N-cadherin (SNAPNcad) and monitored the cell surface spatial dynamics of APP intermediates relative to $\mathrm{N}$-cadherin-based AJs (NAJs) in the presence of protease inhibitors. Having an intermediate ECD size (80 kD), full-length APP showed binary localization (i.e., excluded or enriched) relative to AJs in the presence of inhibitors (Fig. 7a,b and Extended Data Fig. 7a,b), similar to the Notch variant with EGF repeat truncation (i.e., $\mathrm{N} \triangle \mathrm{EGF}$ ). APP diffused into the NAJs after ECD shedding by $\alpha$ - or $\beta$-secretase, and then was processed by $\gamma$-secretase within it (Fig. 7a,b and Extended Data Fig. 7a,b).

APP proteolysis by $\gamma$-secretase produces more soluble $\mathrm{p} 3$ and $A \beta_{40}$ predominately, along with less soluble and pathogenic $A \beta_{42}$ and longer isoforms ${ }^{72,73,75}$. It has been previously shown that local acidic $\mathrm{pH}$ environment (e.g., $\mathrm{pH} 5.5$ ) leads to a gain in the proportion of pathogenic $A \beta$ species ${ }^{76}$. Additionally, $\mathrm{N}$-cadherin expression in cells stabilizes an open conformation of PS1 that favors $A \beta_{40}$ production over 
$A \beta_{42}{ }^{77}$. Given our previous observation that loss of AJs leads to a decrease in cell surface $\gamma$-secretase, we hypothesized that APP processing would be biased under these conditions towards $A \beta_{42}$. We tested this hypothesis by constructing U2OS cell lines lacking both $\mathrm{E}$ - and $\mathrm{N}$-cadherins (CDH1/2-KO cells) using CRISPR-Cas9 (Extended Data Fig. 5a-d). We then transfected plain U2OS cells or CDH1/2-KO cells with a plasmid encoding APP and measured APP fragment production by ELISA. While no significant changes were observed in total $A \beta(40+42)$ and soluble APP $\alpha($ SAPP $\alpha)$ (Fig. 7c,d), CDH1/2-KO cells produced higher relative levels of $A \beta_{42}$, the isoform prone to severe fibril aggregation, compared to cells with endogenous cadherin expression (Fig. 7e).

\section{Discussion}

Unlike most other juxtacrine signaling systems, the Notch ligand-receptor interaction (a binding switch) is converted into intracellular signals only following multiple additional regulatory steps gated by mechanical, enzymatic, and spatial events. These include unfolding of the negative regulatory region (a mechanical switch), S2- and S3- cleavage (proteolytic switches), and finally translocation of the NICD from the cell membrane to the nucleus (a spatial switch) ${ }^{24-26}$. Our study reveals that Notch integrates an additional spatial switch via AJ-driven interfacial membrane compartmentalization to tightly choreograph the critical and irreversible enzymatic cleavage sequence prior to NICD release. Previously, it was thought that this enzymatic sequence was regulated by modification of the molecular interface between Notch and nicastrin after S2-cleavage ${ }^{61,62}$. Our model is not incompatible with a contribution of the nicastrin-Notch chemical interface on $\gamma$-secretase activity. However, it strongly suggests that the AJdriven membrane compartmentalization is the major regulator of Notch- $\gamma$-secretase interaction and signaling, functioning by increasing the concentration of the $\gamma$-secretase substrate to the point that it exceeds the $\mathrm{K}_{\mathrm{M}}$ and is efficiently processed by the enzyme. Particularly, mechanogenetic experiments shown in Fig. 3a,b and Fig. 4i-I, respectively, supports the notion that Notch with an intact S2 site is effectively engaged and then processed so long as the spatial constraint of juxtaposed cell membranes at AJs is removed.

The operating principle of this new spatial switch is closely related to another unique feature of Notch receptor: its unusually tall extracellular domain. The functional residues responsible for ligand binding are located near the $\mathrm{N}$-terminus, which protrudes above the crowded cell surface, where they are poised to engage ligands on neighboring cells. Surprisingly, however, it has also been shown that replacing the EGF-like domain repeats with a smaller ligand binding domain (e.g., synNotch) maintains the receptor function ${ }^{78,79}$. Why then does Notch receptor bear such a massive ECD? Our study provides insight into this question, where the large ECD is crucial for its spatial segregation from $\gamma$-secretase thereby minimizing nonspecific ligand-independent activation. Low level NICD production was observed even for Notch variants with partial EGF truncation (N $\triangle E G_{1-25}$ ) and levels gradually increased upon successive truncations. N $\Delta \mathrm{EGF}_{1-25}$ has comparable size to smaller Notch family homologs, including $\mathrm{C}$. elegans LIN-12/Notch and GLP-1/Notch (13 and 10 EGF repeats, respectively), suggesting the relevance of a spatial switch across the Notch family and metazoans. We also observed mixed distribution of $\mathrm{N} \Delta \mathrm{EGF}$ variant that is smaller than AJ cleft heights. We interpreted that this unanticipated exclusion might result from a lateral crowding effect in high-density AJs, because density of cadherin clusters within AJs varies with the size, type, and degree of junction maturation ${ }^{80,81}$ and the glycosylated negative regulatory region domain ${ }^{82}$ can be susceptible to steric crowding. Our model also explains previous observations where synNotch with a relatively small ECD exhibited significant ligand-independent activation (10-50\% of ligand-induced activation $)^{59}$.

We also show that size-dependent spatial segregation regulates APP cleavage and A production. It has been previously shown that $\gamma$-secretase presenting in different subcellular compartments cleaves APP into diverse $A \beta$ isoforms ${ }^{73,75,83}$. Our study shows that, after the ECD cleavage, AJ potentiates cell surface processing of APPs within the junction, yielding $A \beta_{40}$ predominantly, while removal of $A J$ produces more $A \beta_{42}$. To establish the relevance of this observation to APP processing will require further investigation in a neuronal system, but our results in model cell lines are consistent with the predominant secretion of $A \beta$ from the synapse, where $\mathrm{N}$-cadherin junctions localize ${ }^{84}$. More importantly, these finding suggest that AJ may represent proteolytic hotspots with size-dependent substrate selectivity across a more diverse range of cell-surface proteins. 
Our study also suggests a critical role of the AJ-mediated membrane compartmentalization in VZNPC maintenance and differentiation during development. It has been previously proposed that apicalendfoot AJs promote Notch signaling in NPCs ${ }^{69-71}$, but the mechanism underlying precise Notch signal regulation was unclear. Our findings suggests that Notch signaling is maintained by creating RIP$\mu d o m a i n s$ within AJs, and disruption of the AJs downregulates Notch signaling and hence promotes NPC differentiation. This result also provides important insights on the molecular mechanism of how environmental spatial and physical changes of cells (i.e., VZ-NPC detachment and radial migration) direct cell signaling (i.e., Notch signaling) and differentiation, finally enabling spatiotemporally coordinated tissue development.

The size-dependent segregation of Notch from the RIP- $\mu$ domain has important analogies to the kinetic segregation model of T cell activation, where the large CD45 phosphatase is excluded from T cell receptor (TCR) immunological synaptic clefts ${ }^{18,19,85}$. However, there are several distinct features of the Notch spatial switch compared to the kinetic segregation model. First, unlike the immunological synapse where TCR and CD45 remain constant in size throughout activation, Notch undergoes a dramatic size change during the course of cell surface activation, enabling its dynamic spatial redistribution and sequential proteolysis. Second, the role of AJs in Notch signaling is not limited to creating a physical barrier, but also plays the critical role of recruiting and concentrating $\gamma$-secretase to facilitate processing of S2-cleaved Notch at the cell surface. Third and finally, the consequences of size-dependent segregation on signaling are reversed in comparison to the immunological synapse. While spatial segregation of CD45 enables sustained TCR phosphorylation and downstream signaling, Notch segregation from AJs inhibits signal activation. Our result extends the relevance of size-dependent spatial segregation models beyond immune cells ${ }^{18,19,85,86}$, supporting the notion that size-dependent protein segregation can serve as a general mechanism for regulating a broad range of receptor signaling at the cell-cell interface, including Notch and APPs. It is also important to note that our model may not be limited to the AJs, but may be extended to other cell-cell junctions that provides an environment for sizedependent protein segregation while effectively concentrating proteases.

Overall, AJ-mediated interfacial membrane compartmentalization not only sheds light on the mechanism underlying the sequential proteolysis of Notch and APPs, but also may extend to other receptors processed by $\gamma$-secretase. Finally, we anticipate further implications of our work in other areas of research such as providing new design principles for synthetic receptors like synNotch, as well as new therapeutic approaches that target Notch and APP signaling by spatial mutation in cancer and neurodegenerative diseases.

\section{References}

1. Brasch, J., Harrison, O. J., Honig, B. \& Shapiro, L. Thinking outside the cell: how cadherins drive adhesion. Trends in Cell Biology 22, 299-310 (2012).

2. Honig, B. \& Shapiro, L. Adhesion Protein Structure, Molecular Affinities, and Principles of CellCell Recognition. Cell 181, 520-535 (2020).

3. Harrison, O. J. et al. The Extracellular Architecture of Adherens Junctions Revealed by Crystal Structures of Type I Cadherins. Structure 19, 244-256 (2011).

4. Chen, C. S., Tan, J. \& Tien, J. Mechanotransduction at Cell-Matrix and Cell-Cell Contacts. Annual Review of Bioengineering 6, 275-302 (2004).

5. Yap, A. S., Gomez, G. A. \& Parton, R. G. Adherens Junctions Revisualized: Organizing Cadherins as Nanoassemblies. Developmental Cell 35, 12-20 (2015).

6. Yap, A. S. \& Kovacs, E. M. Direct cadherin-activated cell signaling a view from the plasma membrane. Journal of Cell Biology 160, 11-16 (2003).

7. Manz, B. N. \& Groves, J. T. Spatial organization and signal transduction at intercellular junctions. Nature Reviews Molecular Cell Biology 2010 11:5 11, 342-352 (2010). 
8. Südhof, T. C. Synaptic Neurexin Complexes: A Molecular Code for the Logic of Neural Circuits. Cell 171, 745-769 (2017).

9. Südhof, T. C. \& Malenka, R. C. Understanding Synapses: Past, Present, and Future. Neuron 60, 469-476 (2008).

10. Zeng, M. et al. Reconstituted Postsynaptic Density as a Molecular Platform for Understanding Synapse Formation and Plasticity. Cell 174, 1172-1187.e16 (2018).

11. Sanes, J. R. \& Zipursky, S. L. Synaptic Specificity, Recognition Molecules, and Assembly of Neural Circuits. Cell 181, 536-556 (2020).

12. Davis, D. M. \& Dustin, M. L. What is the importance of the immunological synapse? Trends in Immunology 25, 323-327 (2004).

13. Grakoui, A. et al. The Immunological Synapse: A Molecular Machine Controlling T Cell Activation. Science 285, 221-227 (1999).

14. Narui, Y. \& Salaita, K. Membrane Tethered Delta Activates Notch and Reveals a Role for SpatioMechanical Regulation of the Signaling Pathway. Biophysical Journal 105, 2655-2665 (2013).

15. Schmid, E. M. et al. Size-dependent protein segregation at membrane interfaces. Nature Physics 2016 12:7 12, 704-711 (2016).

16. Salaita, K. et al. Restriction of receptor movement alters cellular response: Physical force sensing by EphA2. Science 327, 1380-1385 (2010).

17. Dustin, M. \& Shaw, A. Costimulation: building an immunological synapse. Science (New York, N.Y.) 283, 649-650 (1999).

18. Groves, J. T. \& Kuriyan, J. Molecular mechanisms in signal transduction at the membrane. Nature Structural \& Molecular Biology 2010 17:6 17, 659-665 (2010).

19. van der Merwe, P. A. \& Dushek, O. Mechanisms for T cell receptor triggering. Nature Reviews Immunology 2011 11:1 11, 47-55 (2010).

20. Belardi, B., Son, S., Felce, J. H., Dustin, M. L. \& Fletcher, D. A. Cell-cell interfaces as specialized compartments directing cell function. Nature Reviews Molecular Cell Biology 2020 21:12 21, 750-764 (2020).

21. Case, L. B., Zhang, X., Ditlev, J. A. \& Rosen, M. K. Stoichiometry controls activity of phaseseparated clusters of actin signaling proteins. Science 363, 1093-1097 (2019).

22. James, J. R. \& Vale, R. D. Biophysical mechanism of T-cell receptor triggering in a reconstituted system. Nature 2012 487:7405 487, 64-69 (2012).

23. Case, L. B., Ditlev, J. A. \& Rosen, M. K. Regulation of Transmembrane Signaling by Phase Separation. Annual Review of Biophysics 48, 465-494 (2019).

24. Bray, S. J. Notch signalling: A simple pathway becomes complex. Nature Reviews Molecular Cell Biology vol. 7 678-689 (2006).

25. Bray, S. J. Notch signalling in context. Nature Reviews Molecular Cell Biology vol. 17 722-735 (2016).

26. Kovall, R. A., Gebelein, B., Sprinzak, D. \& Kopan, R. The Canonical Notch Signaling Pathway: Structural and Biochemical Insights into Shape, Sugar, and Force. Developmental Cell vol. $41228-241$ (2017).

27. Kopan, R. \& Ilagan, M. X. G. The Canonical Notch Signaling Pathway: Unfolding the Activation Mechanism. Cell vol. 137 216-233 (2009).

28. van Es, J. H. et al. Notch/Y-secretase inhibition turns proliferative cells in intestinal crypts and adenomas into goblet cells. Nature 2005 435:7044 435, 959-963 (2005). 
29. S Artavanis-Tsakonas, M. R. R. L. Notch signaling: cell fate control and signal integration in development. Science 284, 770-776 (1999).

30. Boukhatmi, H., Martins, T., Pillidge, Z., Kamenova, T. \& Bray, S. Notch Mediates Inter-tissue Communication to Promote Tumorigenesis. Current Biology 30, 1809-1820.e4 (2020).

31. South, A. P., Cho, R. J. \& Aster, J. C. The double-edged sword of Notch signaling in cancer. Seminars in Cell \& Developmental Biology 23, 458-464 (2012).

32. Nicolas, M. et al. Notch1 functions as a tumor suppressor in mouse skin. Nature Genetics 33, 416-421 (2003).

33. Lai, E. C. Notch signaling: control of cell communication and cell fate. Development 131, 965973 (2004).

34. Haines, N. \& Irvine, K. D. Glycosylation regulates Notch signalling. Nature Reviews Molecular Cell Biology 2003 4:10 4, 786-797 (2003).

35. Stanley, P. \& Okajima, T. Roles of Glycosylation in Notch Signaling. Current Topics in Developmental Biology 92, 131-164 (2010).

36. Wang, X. \& Ha, T. Defining Single Molecular Forces Required to Activate Integrin and Notch Signaling. Science 340, 991-994 (2013).

37. Gordon, W. R. et al. Mechanical Allostery: Evidence for a Force Requirement in the Proteolytic Activation of Notch. Developmental Cell 33, 729-736 (2015).

38. Seo, D. et al. A Mechanogenetic Toolkit for Interrogating Cell Signaling in Space and Time. Cell 165, 1507-1518 (2016).

39. Falo-Sanjuan, J. \& Bray, S. J. Membrane architecture and adherens junctions contribute to strong Notch pathway activation. bioRxiv 2021.05.26.445755 (2021) doi:10.1101/2021.05.26.445755.

40. Guillaume, E. et al. Flotillin microdomains stabilize cadherins at cell-cell junctions. Journal of Cell Science 126, 5293-5304 (2013).

41. Causeret, M., Taulet, N., Comunale, F., Favard, C. \& Gauthier-Rouvière, C. N-Cadherin Association with Lipid Rafts Regulates Its Dynamic Assembly at Cell-Cell Junctions in C2C12 Myoblasts. Molecular Biology of Cells 16, 2168-2180 (2005).

42. Taulet, N. et al. N-cadherin/p120 Catenin Association at Cell-Cell Contacts Occurs in Cholesterolrich Membrane Domains and Is Required for RhoA Activation and Myogenesis. Journal of Biological Chemistry 284, 23137-23145 (2009).

43. Szaruga, M. et al. Alzheimer's-Causing Mutations Shift $A \beta$ Length by Destabilizing $Y$-SecretaseAßn Interactions. Cell 170, 443-456.e14 (2017).

44. Lee, S.-J. et al. A detergent-insoluble membrance compartment contains $A \beta$ in vivo. Nature Medicine 1998 4:6 4, 730-734 (1998).

45. Vetrivel, K. S. et al. Spatial Segregation of Y-Secretase and Substrates in DistinctMembraneDomains. Journal of Biological Chemistry 280, 25892-25900 (2005).

46. Chow, V. W., Mattson, M. P., Wong, P. C. \& Gleichmann, M. An Overview of APP Processing Enzymes and Products. NeuroMolecular Medicine 2009 12:1 12, 1-12 (2009).

47. Ehehalt, R., Keller, P., Haass, C., Thiele, C. \& Simons, K. Amyloidogenic processing of the Alzheimer $\beta$-amyloid precursor protein depends on lipid rafts. Journal of Cell Biology 160, 113-123 (2003).

48. Wahrle, S. et al. Cholesterol-Dependent $\mathrm{y}$-Secretase Activity in Buoyant Cholesterol-Rich Membrane Microdomains. Neurobiology of Disease 9, 11-23 (2002).

49. Vetrivel, K. S. et al. Association of y-Secretase with Lipid Rafts in Post-Golgi and Endosome Membranes. Journal of Biological Chemistry 279, 44945-44954 (2004). 
50. Urano, Y. et al. Association of active y-secretase complex with lipid rafts. Journal of Lipid Research 46, 904-912 (2005).

51. Galbiati, F., Razani, B. \& Lisanti, M. P. Emerging Themes in Lipid Rafts and Caveolae. Cell 106, 403-411 (2001).

52. Raghupathy, R. et al. Transbilayer Lipid Interactions Mediate Nanoclustering of Lipid-Anchored Proteins. Cell 161, 581-594 (2015).

53. Kim, J., Jeong, H., Southard, K. M., Jun, Y. \& Cheon, J. Magnetic Nanotweezers for Interrogating Biological Processes in Space and Time. Accounts of Chemical Research 51, 839-849 (2018).

54. Kwak, M. et al. Small, Clickable, and Monovalent Magnetofluorescent Nanoparticles Enable Mechanogenetic Regulation of Receptors in a Crowded Live-Cell Microenvironment. Nano Letters 19, 3761-3769 (2019).

55. Luca, V. C. et al. Structural basis for Notch1 engagement of Delta-like 4. Science 347, 847-853 (2015).

56. Polacheck, W. J. et al. A non-canonical Notch complex regulates adherens junctions and vascular barrier function. Nature 552, 258-262 (2017).

57. Struhl, G. \& Adachi, A. Requirements for Presenilin-Dependent Cleavage of Notch and Other Transmembrane Proteins. Molecular Cell 6, 625-636 (2000).

58. Palmer, W. H. \& Deng, W.-M. Ligand-Independent Mechanisms of Notch Activity. Trends in Cell Biology 25, 697-707 (2015).

59. Yang, Z., Yu, Z., Cai, Y., Du, R. \& Cai, L. Engineering of an enhanced synthetic Notch receptor by reducing ligand-independent activation. Communications Biology 2020 3:1 3, 1-7 (2020).

60. Mumm, J. S. et al. A Ligand-Induced Extracellular Cleavage Regulates Y-Secretase-like Proteolytic Activation of Notch1. Molecular Cell 5, 197-206 (2000).

61. Bolduc, D. M., Montagna, D. R., Gu, Y., Selkoe, D. J. \& Wolfe, M. S. Nicastrin functions to sterically hinder $\mathrm{Y}$-secretase-substrate interactions driven by substrate transmembrane domain. Proceedings of the National Academy of Sciences 113, E509-E518 (2016).

62. Shah, S. et al. Nicastrin Functions as a y-Secretase-Substrate Receptor. Cell 122, 435-447 (2005).

63. Liang, S. I. et al. Phosphorylated EGFR Dimers Are Not Sufficient to Activate Ras. Cell Reports 22, 2593-2600 (2018).

64. Sprinzak, D. et al. Cis-interactions between Notch and Delta generate mutually exclusive signalling states. Nature 465, 86-90 (2010).

65. Nandagopal, N. et al. Dynamic Ligand Discrimination in the Notch Signaling Pathway. Cell 172, 869-880.e19 (2018).

66. Ables, J. L., Breunig, J. J., Eisch, A. J. \& Rakic, P. Not(ch) just development: Notch signalling in the adult brain. Nature Reviews Neuroscience 12, 269-283 (2011).

67. A Louvi, S. A.-T. Notch signalling in vertebrate neural development. Nature Rev. Neurosci. 7, 93102 (2006).

68. Franco, S. J. \& Müller, U. Shaping Our Minds: Stem and Progenitor Cell Diversity in the Mammalian Neocortex. Neuron 77, 19-34 (2013).

69. Das, R. M. \& Storey, K. G. Apical Abscission Alters Cell Polarity and Dismantles the Primary Cilium During Neurogenesis. Science 343, 200-204 (2014).

70. Fujita, I. et al. Endfoot regeneration restricts radial glial state and prevents translocation into the outer subventricular zone in early mammalian brain development. Nature Cell Biology 2019 22:1 22, 2637 (2019). 
71. Hatakeyama, J. et al. Cadherin-based adhesions in the apical endfoot are required for active Notch signaling to control neurogenesis in vertebrates. Development 141, 1671-1682 (2014).

72. O'Brien, R. J. \& Wong, P. C. Amyloid Precursor Protein Processing and Alzheimer's Disease. Annual review of Neuroscience 34, 185-204 (2011).

73. Selkoe, D. J. \& Hardy, J. The amyloid hypothesis of Alzheimer's disease at 25 years. EMBO Molecular Medicine 8, 595-608 (2016).

74. Pepys, M. B. Amyloidosis. Annual Review of Medicine 57, 223-241 (2006).

75. de Strooper, B. \& Karran, E. The Cellular Phase of Alzheimer's Disease. Cell 164, 603-615 (2016).

76. Quintero-Monzon, O. et al. Dissociation between the Processivity and Total Activity of $\mathrm{Y}^{-}$ Secretase: Implications for the Mechanism of Alzheimer's Disease-Causing Presenilin Mutations. Biochemistry 50, 9023-9035 (2011).

77. Uemura, K. et al. N-cadherin-based adhesion enhances $A \beta$ release and decreases $A \beta 42 / 40$ ratio. Journal of Neurochemistry 108, 350-360 (2009).

78. Morsut, L. et al. Engineering Customized Cell Sensing and Response Behaviors Using Synthetic Notch Receptors. Cell 164, 780-791 (2016).

79. Roybal, K. T. et al. Precision Tumor Recognition by T Cells With Combinatorial Antigen-Sensing Circuits. Cell 164, 770-779 (2016).

80. Indra, I. et al. Spatial and temporal organization of cadherin in punctate adherens junctions. Proceedings of the National Academy of Sciences 115, E4406-E4415 (2018).

81. Takeichi, M. Dynamic contacts: rearranging adherens junctions to drive epithelial remodelling. Nature Reviews Molecular Cell Biology 2014 15:6 15, 397-410 (2014).

82. Takeuchi, H. \& Haltiwanger, R. S. Role of glycosylation of Notch in development. Seminars in Cell \& Developmental Biology 21, 638-645 (2010).

83. Yang, G. et al. Structural basis of Notch recognition by human y-secretase. Nature 2018 565:7738 565, 192-197 (2018).

84. Yuksel, M. \& Tacal, O. Trafficking and proteolytic processing of amyloid precursor protein and secretases in Alzheimer's disease development: An up-to-date review. European Journal of Pharmacology 856, 172415 (2019).

85. Dustin, M. L. \& Groves, J. T. Receptor Signaling Clusters in the Immune Synapse. Annual Review of Biophysics 41, 543-556 (2012).

86. Bakalar, M. H. et al. Size-Dependent Segregation Controls Macrophage Phagocytosis of Antibody-Opsonized Targets. Cell 174, 131-142.e13 (2018). 

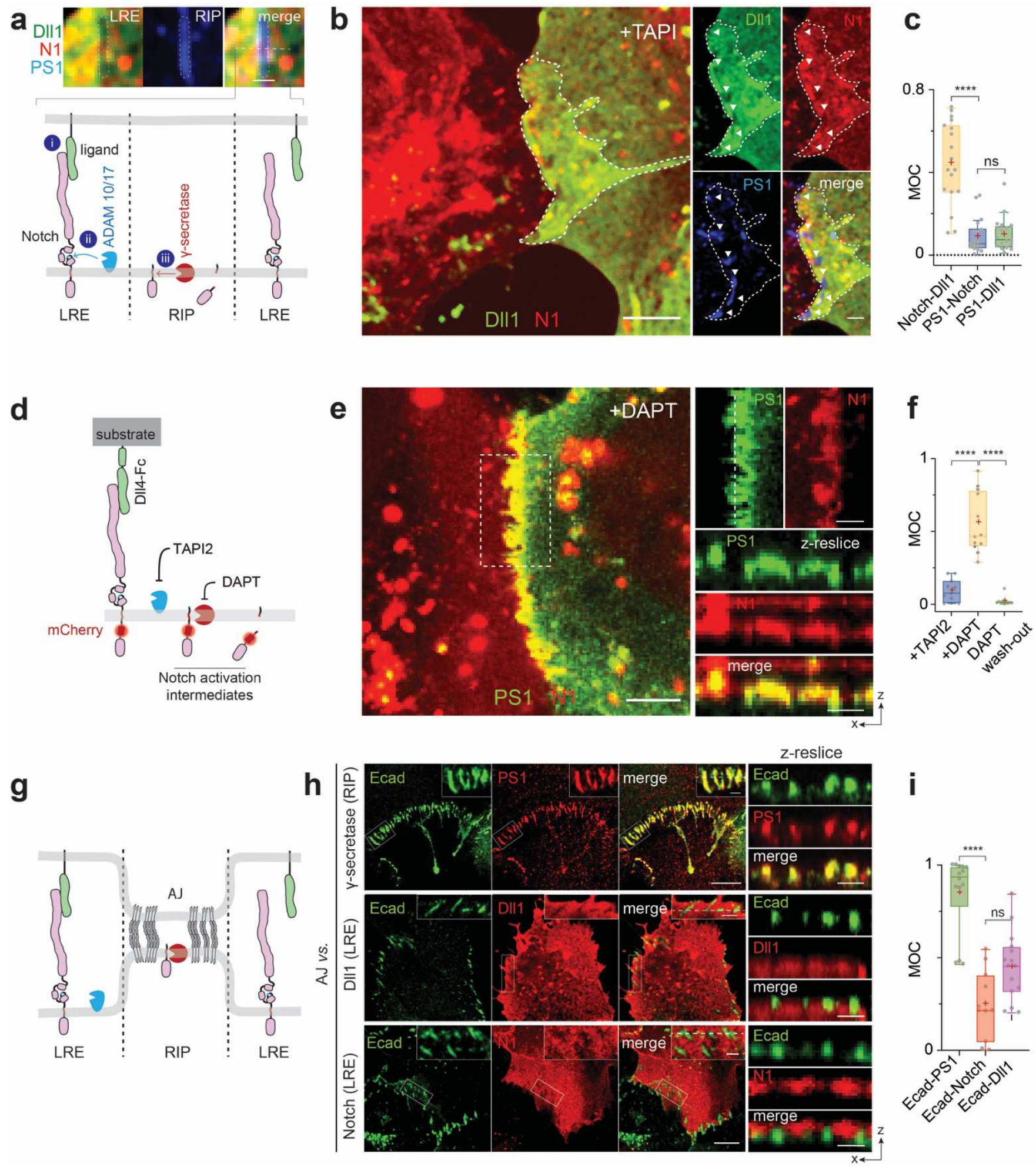

Fig. 1. Interfacial membrane compartmentalization organized by cadherin-based adherens junctions (AJs) creates two discrete microenvironments for the sequential molecular processing of Notch. (a) A schematic showing membrane compartmentalization choreographing the sequential steps in cell-surface activation of Notch. LRE and RIP represent distinct membrane microdomains ( $\mu$ domains) for Notch receptor-ligand engagement and regulated intramembrane proteolysis, respectively. (top) A representative image showing LRE and RIP $\mu$-domains. Scale bar, $2 \mu \mathrm{m}$. (b) Representative confocal fluorescence images showing DII1, Notch1 (N1), and presenilin1 (PS1) distributions at the interfacial membrane formed between cells co-expressing Notch1 and Dll1 in the presence of TAPI2. The area with a white dashed line indicates the cellular interface. (left) A maximum projection image of DII1 and Notch1 constituting a LRE $\mu$ domain. Scale bar, $5 \mu \mathrm{m}$. (right) Individual fluorescent channel and 
merged images for DIl1, Notch1, and presenilin1. Scale bar, $2 \mu \mathrm{m}$. (c) Manders' overlap coefficients (MOCs) for quantitative assessment of Notch with DIl1, presenilin1 with Notch1, and presenilin1 with DII1, respectively. (d) A schematic to capture the spatial distribution of Notch intermediates during the cellsurface activation pathway. (e) Representative confocal images showing strong enrichment of Notch mCherry signal within the RIP $\mu$ domains from the cells activated by culturing them on a DII4-Fc immobilized substrate in the presence of DAPT. (left) A maximum projection image of the cells showing enriched Notch mCherry signal at the RIP $\mu$ domains. Scale bar, $10 \mu \mathrm{m}$. (Top-right) Magnified individual fluorescence channel images of the boxed region. Scale bar, $4 \mu \mathrm{m}$. (right) Z-resliced images showing the sections of the cellular interfaces. Scale bar, $4 \mu \mathrm{m}$. (f) Manders' overlap coefficients for quantitative assessment of Notch1 with presenilin1 during sequential molecular processing of Notch1. (g) A schematic showing AJ-mediated membrane compartmentalization that creates LRE- and RIPmicrodomains. (h) Representative confocal fluorescence images showing spatial distributions of RIP(presenilin1) and LRE- (DII1 and Notch1) $\mu$ domains relative to E-cadherin-based adherens junctions (AJs). (left) A maximum projection image. Inset shows a magnified image of the boxed area highlighting the membrane microdomains at cellular interfaces. Scale bar, $2 \mu \mathrm{m}$. (right) z-resliced images. Scale bar, $2 \mu \mathrm{m}$. (i) Manders' overlap coefficients for quantitative assessment of PS1, DIl1, and Notch1 localization with E-cadherin. (c, f, and i) Overlays are box and whisker plots; ${ }^{* * * * P}<0.0001$, ns: non-significant; oneway ordinary ANOVA followed by Tukey's multiple comparison testing. 
a
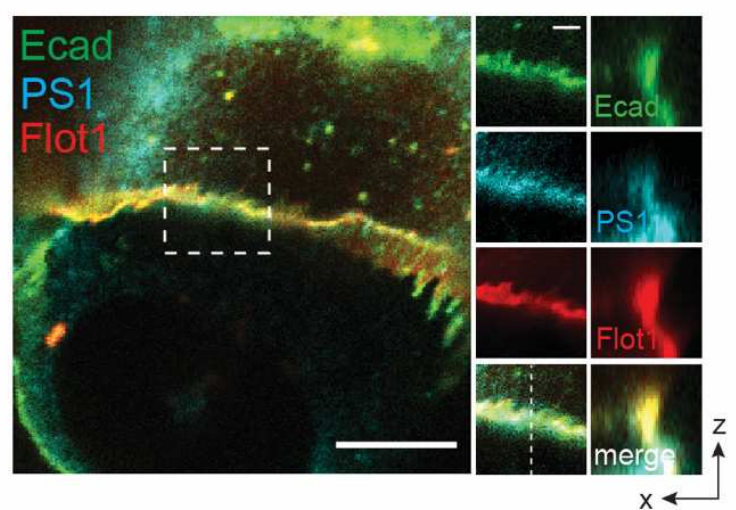

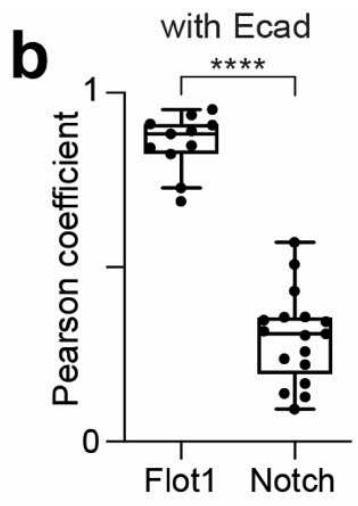

C no DPPS with $30 \%$ DPPS

C immobilzation immobilzation

\section{के 들 \\ 㐫}
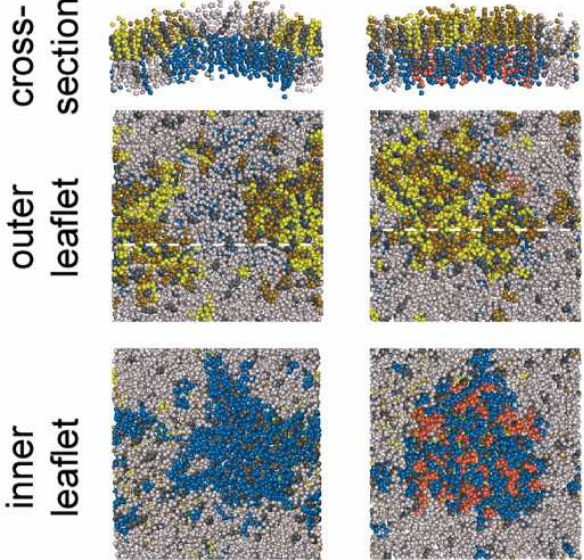

d

$\square$ no immobilization $\square 30 \%$ immobilization
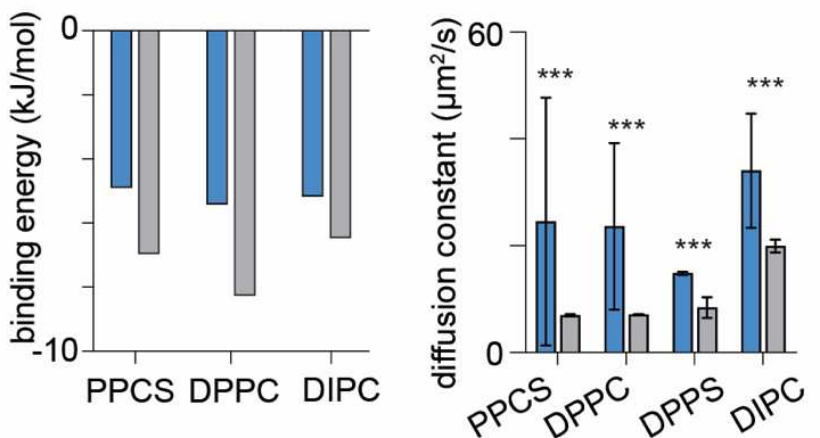

f

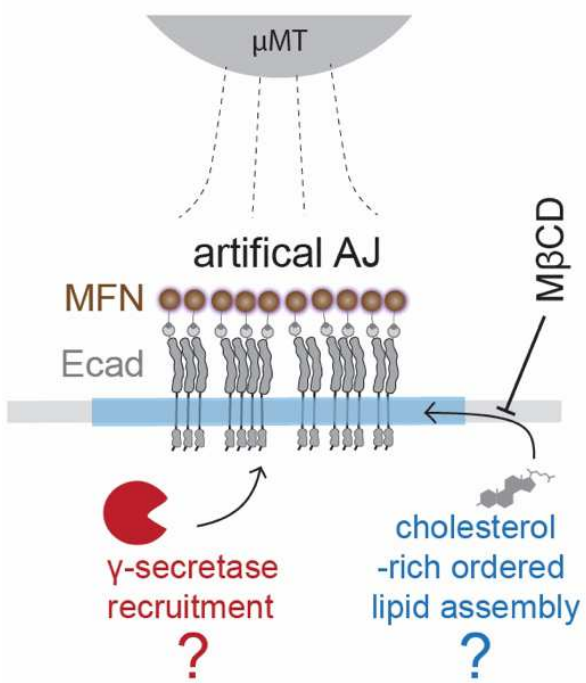

d

e 


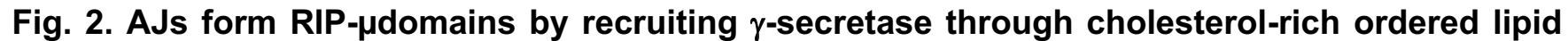
assemblies. (a) Representative confocal fluorescence images showing the PS1 and Flot1 distribution relative to native cell-cell AJs. (left) A maximum projection image of merged channels. Scale bar, $10 \mu \mathrm{m}$. (center) Magnified images of the boxed region. Scale bar, $2 \mu \mathrm{m}$. (right) z-resliced images showing the sections of the AJs. Scale bar, $2 \mu \mathrm{m}$. (b) Manders' overlap coefficients for quantitative assessment of Flot1 and Notch colocalization with E-cadherin. Overlaid are box and whisker plots. ${ }^{* * * *} P<0.0001$. Twotailed unpaired Student's t test. (c) Snapshot images of coarse-grained MD simulation of a lipid bilayer comprising of 1,2-dilinoleoyl-sn-glycero-3-phosphocholine (DIPC; gray), 1,2-dipalmitoyl-sn-glycero-3phosphocholine (DPPC; yellow), N-palmitoyl-O-phosphocholineserine (PPCS; green), 1,2-dipalmitoylsn-glycero-3-phosphoserine (DPPS; blue), immobilized DPPS (pink), and cholesterol (CHOL; grey). Left and right panels represent the simulation results without and with a partial (30\%) DPPS immobilization, respectively. (d, e) Diffusion coefficients (c) and binding energy (d) of individual lipid components in a lipid bilayer with or without a partial (30\%) DPPS immobilization in MD simulation shown in Fig. 2c. (f) A schematic showing mechanogenetic interrogation of $\gamma$-secretase and cholesterol-rich ordered lipid microassemblies relative to the artificial AJs. Artificial AJs were formed by clustering Ecad-GFP labeled with magnetofluorescent nanoparticles (MFNs) by application of an external micromagnetic tweezer $(\mu \mathrm{MT}) . \mathrm{M} \beta C D$ was used for cholesterol depletion in the cell membrane before artificial AJ formation. (g) Epifluorescence images showing the formation of an artificial AJ by mechanogenetics. After stimulation by $\mu \mathrm{MT}$, vivid MFN and E-cadherin signals at the magnetic focus were seen, indicating formation of AJs. Scale bar, $5 \mu \mathrm{m}$. (h) Confocal fluorescence images of E-cadherin, presenilin1, and Flotillin-1 (Flot1) at the artificial AJ with and without M $\beta C D$ treatment. After cholesterol depletion, no PS1 recruitment was seen at the AJ, suggesting that $\gamma$-secretase recruitment to the AJ requires lipid microdomain formation at the AJ. Scale bar, $2 \mu \mathrm{m}$. 
a

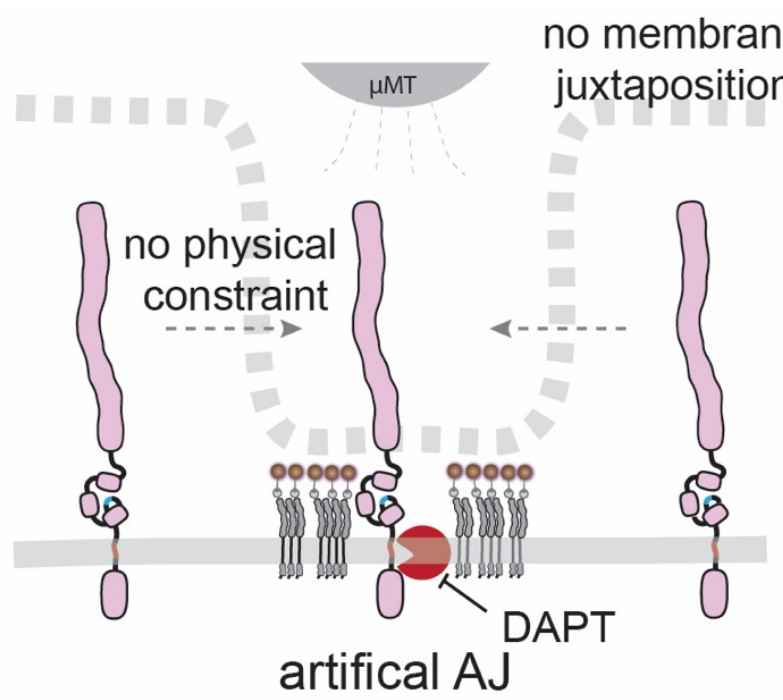

b
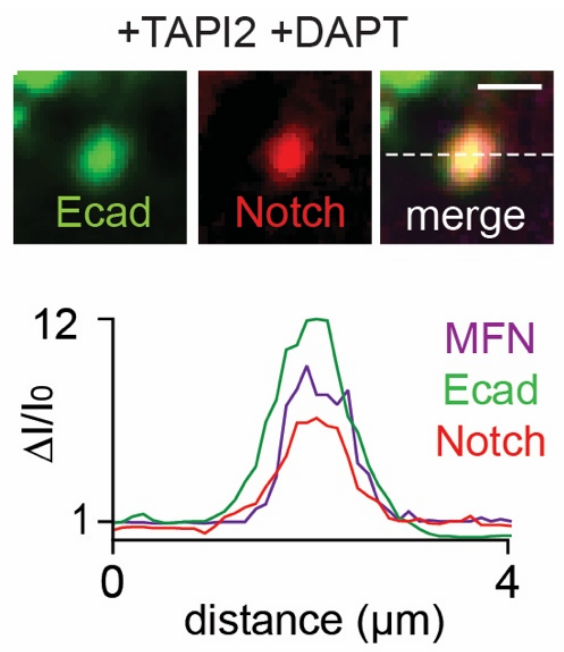

C

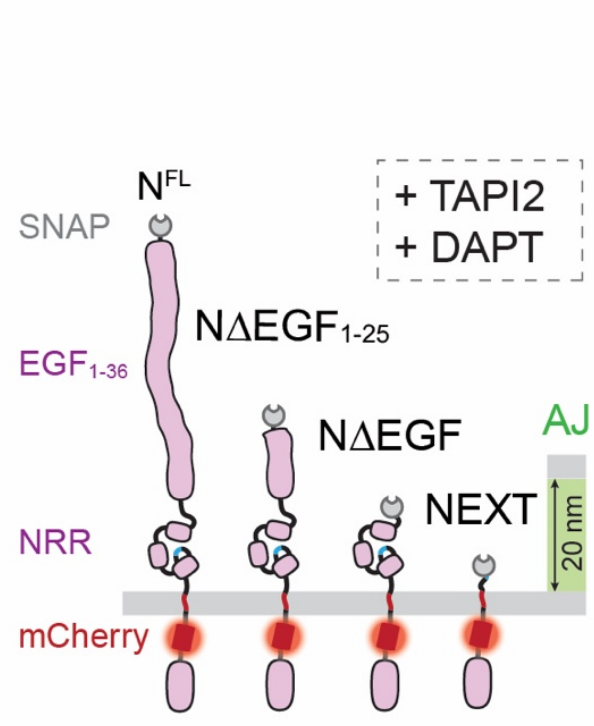

d

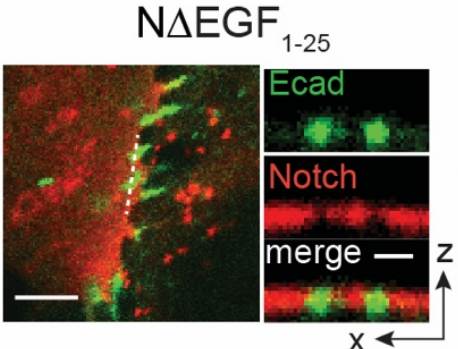

$\mathrm{N} \Delta \mathrm{EGF}$
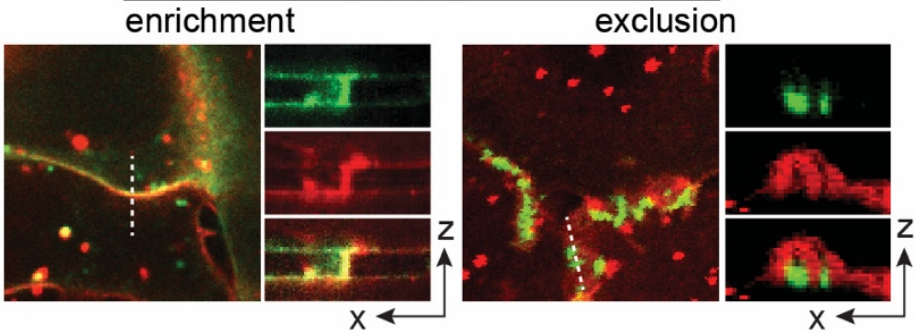

e

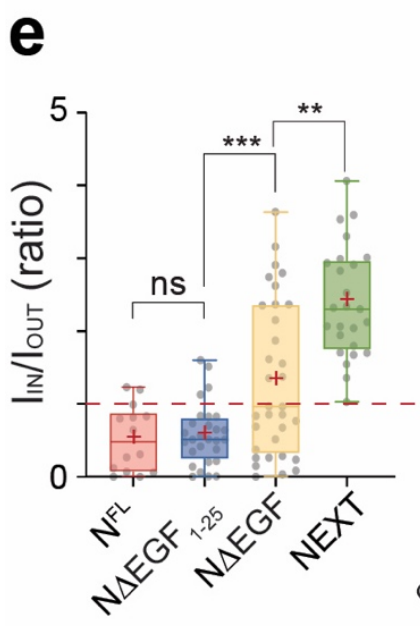

$\mathrm{N} \Delta \mathrm{EGF}$

f g

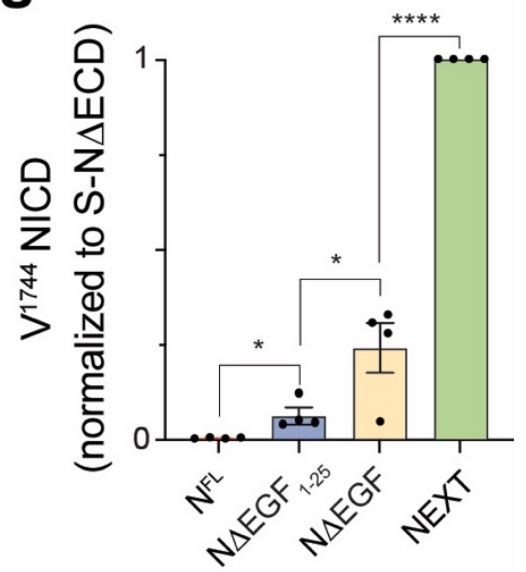

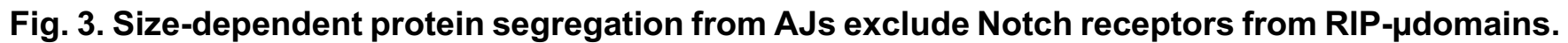
(a) A schematic showing mechanogenetic interrogation of Notch distribution relative to the artificial AJ. Cells treated with DAPT to inhibit $\gamma$-secretase activity. (b) Confocal fluorescence images showing Ecadherin and Notch distributions at the artificial AJs after $\mu \mathrm{MT}$ application. Strong accumulation of Notch signal at the artificial AJ was clearly seen. Line profiles of MFN, E-cadherin, and Notch signals along a 
white dashed line. $\Delta \mathrm{l} / \mathrm{I}_{0}$ represents a fold change relative to fluorescence intensity before stimulation. Scale bar, $2 \mu \mathrm{m}$. (c) Schematics of Notch variants with different truncation lengths, in comparison with the AJ intermembrane cleft. All cells were treated with TAPI2 and DAPT to prevent any potential proteolysis. (d) Confocal fluorescence images showing spatial distribution of the Notch variants (red) relative to the AJs (green). To prevent any ligand-independent activation, cells were incubated with TAPI2 and DAPT. (left) Maximum projections of confocal z-stacks. Scale bar, $5 \mu \mathrm{m}$. (right) Confocal $z$ resliced images along the white dashed lines in the maximum projection images. Scale bar, $2 \mu \mathrm{m}$. (e) Quantification of the enrichment factor $\left(\mathrm{I}_{\mathrm{IN}} / \mathrm{l}_{\mathrm{OUT}}\right)$ of Notch variants relative to the AJs. A box-plot showing binary localization of N $\Delta E G F$ which is defined as either excluded (yellow) or enriched (orange) is shown on the right. Colored lines and $(+)$ marks indicate median and mean, respectively. $(\mathbf{f}, \mathbf{g})$ Western blot analyses of cleaved NICD levels in the cells stably expressing $N^{\mathrm{FL}}, \mathrm{N} \Delta \mathrm{EGF}_{1-25}, \mathrm{~N} \triangle \mathrm{EGF}$, and NEXT. All cells were transfected with Ecad-GFP and incubated with TAPI2 for $24 \mathrm{hr}$. $\beta$-actin levels represent the loading control. A representative image of immunoblotting (f), and quantification (g) of cleaved NICD levels. The average intensity of each NICD band relative to respective $\beta$-actin band was quantified and then normalized to that of NEXT (mean \pm s.d.; $n=4$ biological replicates). $(\mathbf{e}-\mathbf{g}){ }^{*} P<0.05,{ }^{* *} P<0.01$, ${ }^{* * *} \mathrm{P}<0.001,{ }^{* * * *} \mathrm{P}<0.0001$, ns: non-significant; one-way ANOVA followed by Tukey's multiple comparison test. 
a

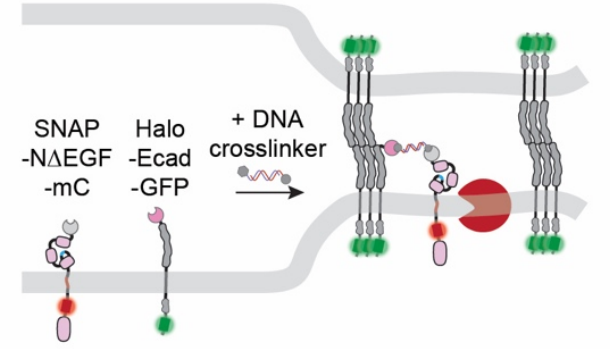

b

d
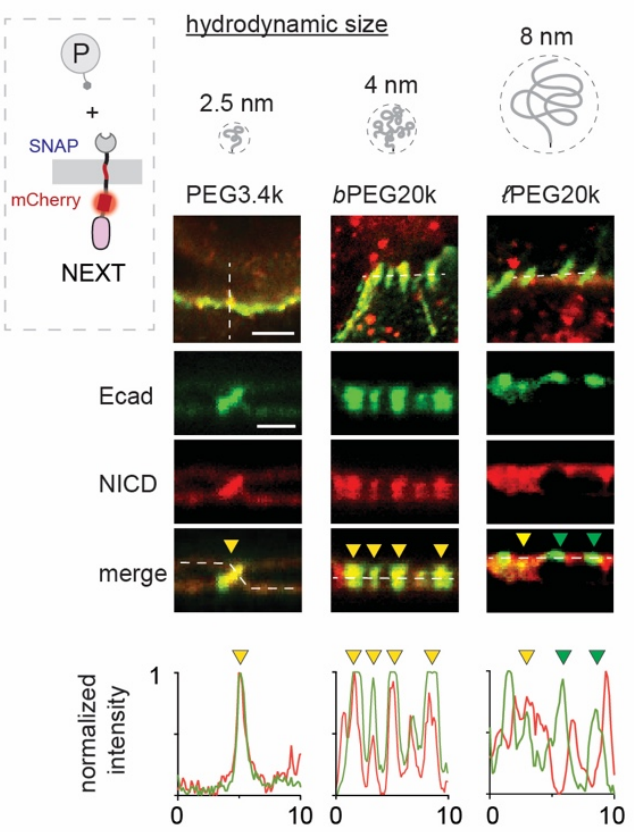
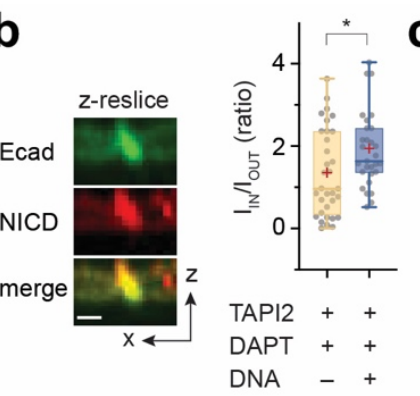

C NICD

$\beta$-acti
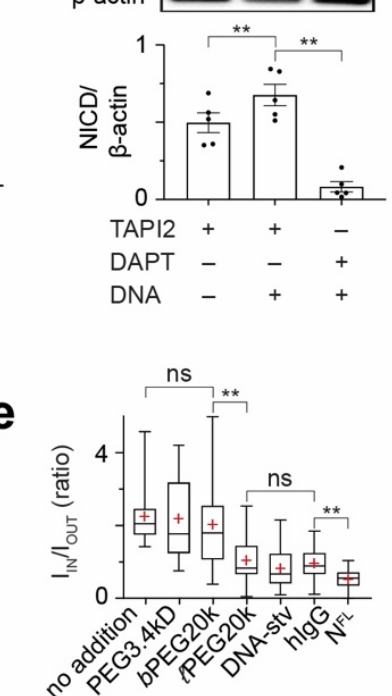

g
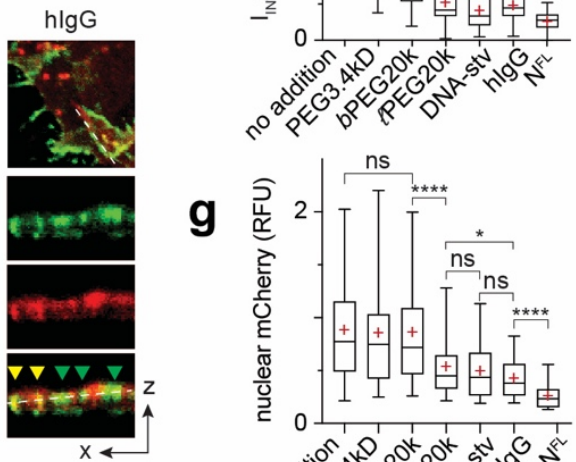

distance $(\mu \mathrm{m})$

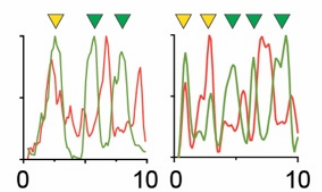

f
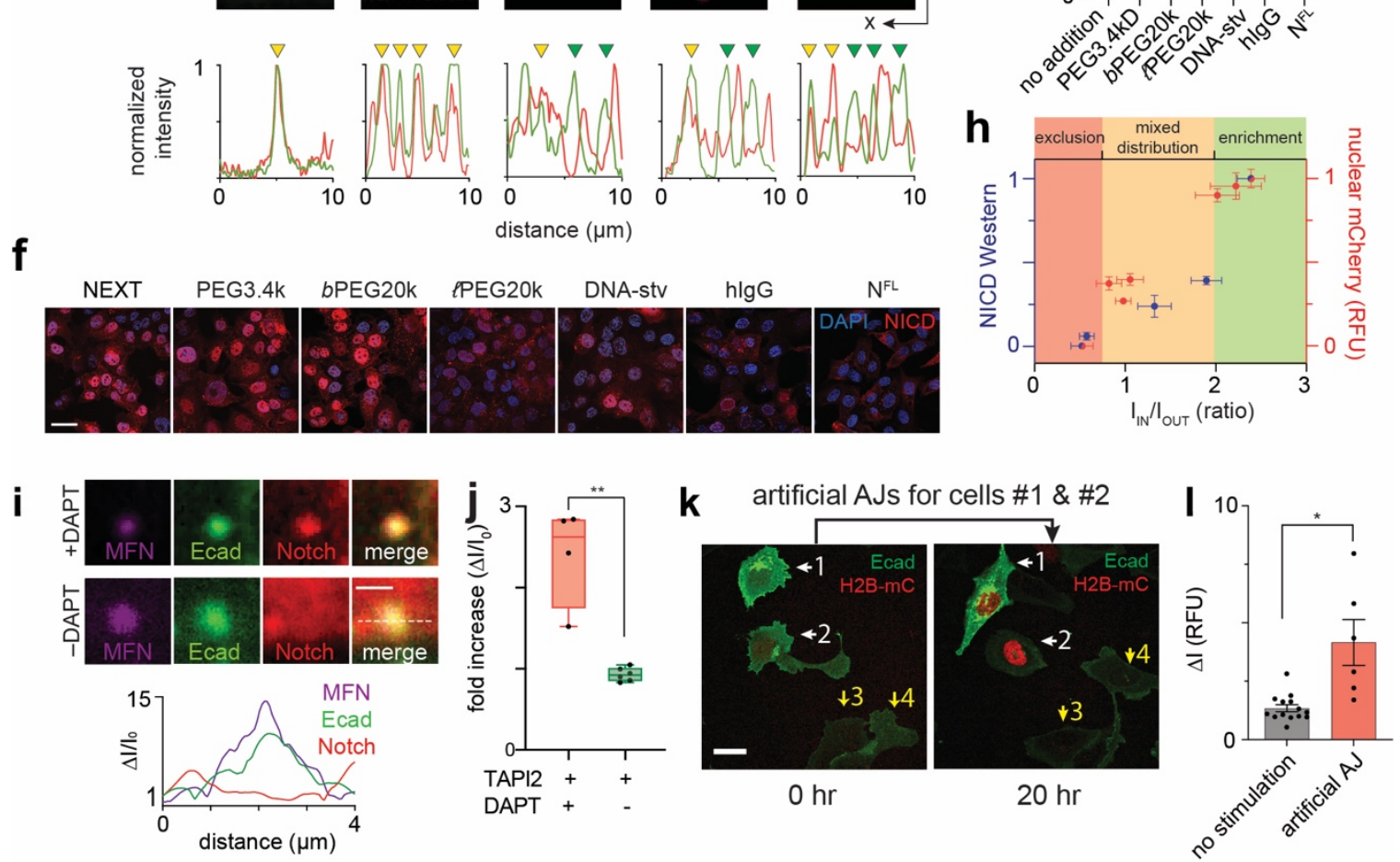

Fig. 4. Spatial mutations alter Notch activation, regardless of ligand presentation or S2 cleavage. (a) A schematic describing DNA-mediated crosslinking strategy to enhance N $\triangle E G F$ localization at the AJ. (b) Confocal z-resliced images showing intense N $\triangle E G F$ fluorescence (red) enriched at the AJ (green) after the DNA crosslinking. Scale bar, $3 \mu \mathrm{m}$. Quantification of enrichment $\left(\mathrm{l}_{\mathrm{IN}} / \mathrm{lout}_{\mathrm{OUT}}\right)$ without $(\mathrm{n}=33)$ and with $(n=29)$ DNA crosslinker treatment, indicating an increase in enrichment after the crosslinking $\left({ }^{*} P<\right.$ 0.05; two-tailed Mann-Whitney-Wilcoxon test). (c) Western blot analyses showing increased S3-cleaved 
NICD levels in the N $\triangle E G F$ cells treated with the DNA crosslinker. (top) A representative gel image showing immunoblotting for NICD and $\beta$-actin. (bottom) Quantification of cleaved NICD levels. The average intensity of NICD band was normalized to that of $\beta$-actin band in each sample. (mean $\pm \mathrm{s}$.d.; ${ }^{* *} \mathrm{P}$ $<0.01 ; n=5$ biological replicates; ordinary one-way ANOVA). (d) Spatial mutation of NEXT via chemical ligation of macromolecular pendants (denoted as P). BG-modified polymers or proteins were conjugated to the extracellular SNAP tag of NEXT. Cartoons depicting shape and hydrodynamic size of different pendants are shown. Confocal images showing size-dependent spatial mutation of NEXT (red) at the AJs (green). The top row shows maximum projection images of the cells treated with the indicated pendants. Scale bar, $5 \mu \mathrm{m}$. The middle row shows confocal $\mathrm{z}$ resliced images along the white dashed lines in the maximum projection images. Yellow and green arrowheads indicate the AJs enriches with and those that excludes Notch, respectively. Scale bar, $3 \mu \mathrm{m}$. The bottom row shows line profiles quantifying fluorescence signals from NEXT (red) and E-cadherin (green) along the white lines in the zresliced images. Images and line profiles are representative of $n \geq 15$ biological replicates. (e) Quantification in $I_{\mathbb{N}} / l_{\text {OUT }}$ of NEXT with macromolecular pendants ( $n \geq 15$ ). (F and $\mathbf{G}$ ) Confocal fluorescence images (f) and quantification $(n \geq 180)(\mathbf{g})$ of nuclear mCherry signal for the NEXTexpressing cells treated with macromolecular pendants. DAPI signal (blue) indicate cell nucleus. Scale bar, $5 \mu \mathrm{m}$. Cells expressing $\mathrm{N}^{\mathrm{FL}}$ were used as a negative control. (h) A plot representing the NICD level of various Notch variants as a function of the enrichment factor ( $\mathrm{I}_{\mathrm{N}} / \mathrm{l}_{\mathrm{OUT}}$, mean \pm s.e.m.; $\mathrm{n} \geq 15$ biological replicates). All Notch variants with different truncation length, DNA crosslinking, or pendant addition used in Fig. 2 and 3 were included. median \pm s.e.m.; $n \geq 4$ for western blot for NICD levels; $n \geq 180$ for nuclear mCherry fluorescence. (i) Representative confocal fluorescence images of cells with an artificial AJ in the presence of TAPI2 and DAPT (upper), and TAPI2 only (lower). Line profiles of MFNs, E-cadherin, and Notch signal along the white dashed line. $\Delta \mathrm{I} / \mathrm{I}_{0}$ represents a fold change relative to nonjunctional membrane signal. Scale bar, $2 \mu \mathrm{m}$. (j) Quantification of mCherry signal at artificial AJs after $\mu$ MT application in the presence or absence of DAPT (with DAPT, $n=4$; no DAPT, $n=6)$. (e, g, j) Boxes and whiskers indicate the interquartile and the full ranges, respectively. Black lines and (+) marks indicate median and mean, respectively. ${ }^{* *} \mathrm{P}<0.01$; ${ }^{* * *} \mathrm{P}<0.001$; ns, not significant; ordinary one-way ANOVA followed by Tukey's multiple comparison. (k) Representative confocal fluorescence images of the reporter cells with artificial AJs. White arrows: cells with stimulation, Yellow arrows: control cells. Scale bar, $10 \mu \mathrm{m}$. (L) Statistical analysis of stimulated cells $(n=6)$ vs. control cells $(n=14)$. Error bars indicate SEM. *P < 0.05; ns, not significant; ordinary one-way ANOVA. 
a group of cells - AJ-.-.

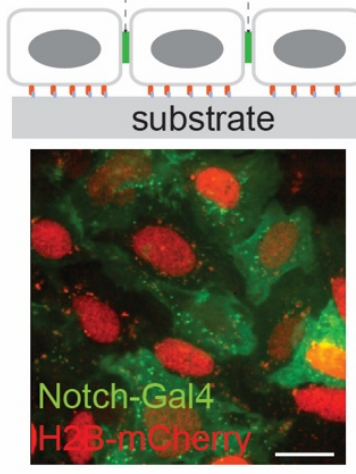

b

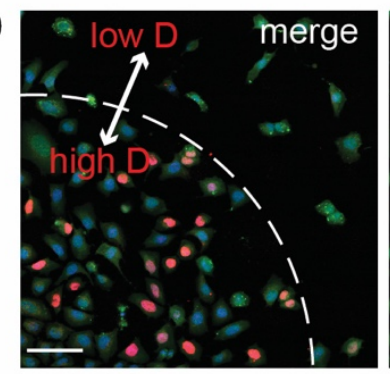

e

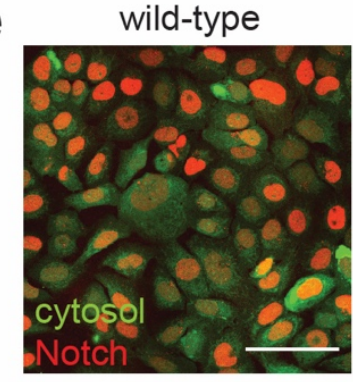

solitary cells
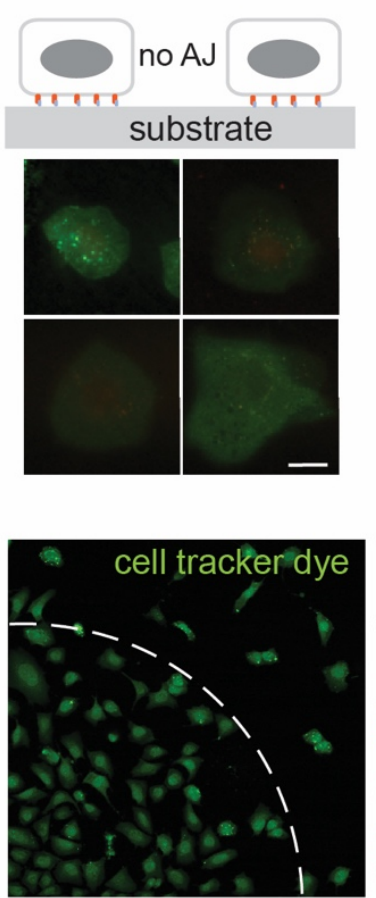

Ecad KO solitary cells on a

Ecad/DII4 substrate
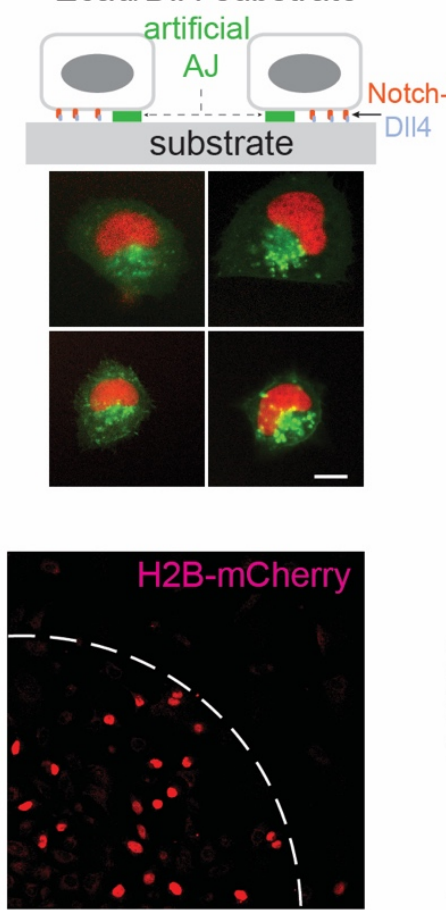

Ecad KO

+ Ecad-GFP

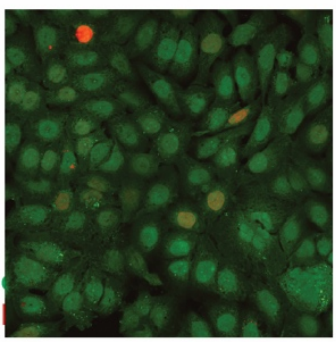

C
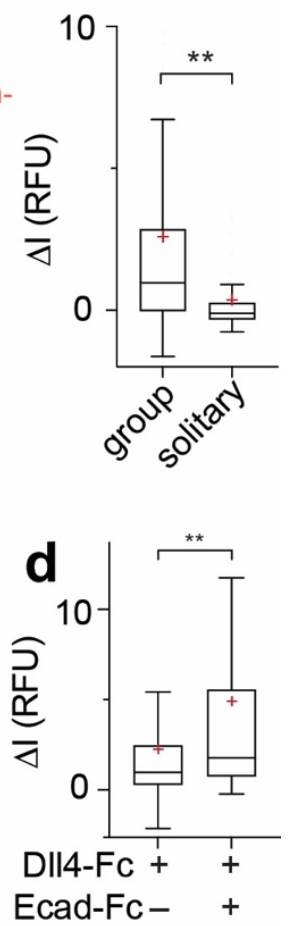

Ecad KO

+ Ncad-GFP $\mathbf{f}$

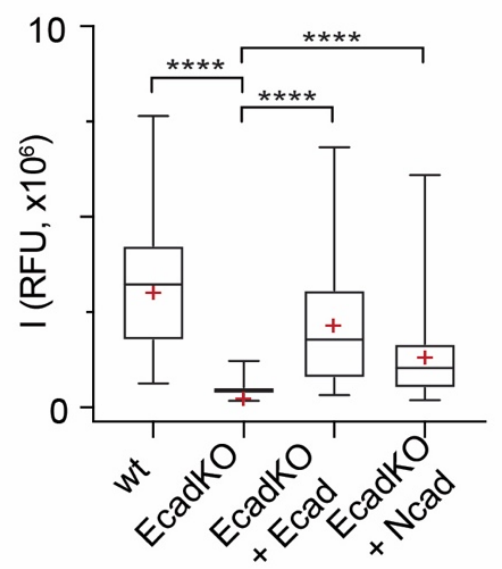

g

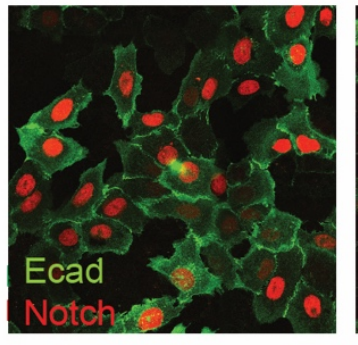

Fig. 5. The AJ-mediated spatial switch regulates Notch signaling. (a) Representative epifluorescence images showing Notch activation in U2OS SNAP-N ${ }^{\mathrm{FL}}$-Gal4 reporter cell lines in different cellular environments: Group of cells on a Dll4-Fc coated substrate (left), solitary cells with no prior contact on a DII4-Fc coated substrate (middle), and solitary cells plated on a DII4-Fc and Ecad-Fc coated substrate (right). Scale bars, $20 \mu \mathrm{m}$ (see Video S1 and S2). (b) Representative low magnification epifluorescence image showing both grouped cells and multiple solitary cells. Scale bar, $100 \mu \mathrm{m}$. (c) Quantification of Notch activation by measuring H2B-mCherry fluorescence changes in cells within a 
group ( $n=152)$, solitary cells $(n=50)$. (d) Quantification of Notch activation in solitary cells cultured on a DII4-Fc coated substrate $(n=27)$ and those cultured on a DII4-Fc and Ecad-Fc coated substrate $(n=$ 27). (e) Representative confocal images of H2B-mCherry fluorescence in U2OS SNAP-N ${ }^{\mathrm{FL}}$-Gal4 reporter cells (wt), E-cadherin knockout cells (Ecad-KO), Ecad-KO cells with recombinant E-cadherin transfection (Ecad-KO + Ecad), and Ecad-KO cells with N-cadherin transfection (Ecad-KO + Ncad). Cytosol labeled with CMFDA dye was shown for wt and Ecad-KO cells. E-cadherin and N-cadherin were shown for Ecad$\mathrm{KO}+$ Ecad and Ecad-KO + Ncad cells. Scale bar, $100 \mu \mathrm{m}$. (f) Quantification of Notch activation in the wt $(n=86)$, Ecad-KO ( $n=100)$, Ecad-KO + Ecad $(n=52)$, and Ecad-KO + Ncad $(n=80)$ cells. $(\mathbf{c}$, $\mathbf{d}$, and f) Boxes and whiskers indicate the interquartile and full ranges, respectively. Black lines and $(+)$ marks indicate median and mean, respectively. ${ }^{*} \mathrm{P}<0.05$; ${ }^{* *} \mathrm{P}<0.01$; ${ }^{* * *} \mathrm{P}<0.001$; ns, not significant; unpaired two-tailed t test in (c) and (d); ordinary one-way ANOVA followed by Tukey's multiple comparison in (f). (g) Comparison of Notch signal activation, readout by mean nuclear H2B-mCherry fluorescence, as a function of E-cadherin expression, readout by membrane GFP fluorescence signal. Each dot represents H2B-mCherry signal of a single cell, and cells are grouped into bins based on their levels of Ecad expression. ${ }^{*} p<0.05,{ }^{* *} p<0.001$, ns, non-significant. One-way ANOVA followed by Tukey's test. 

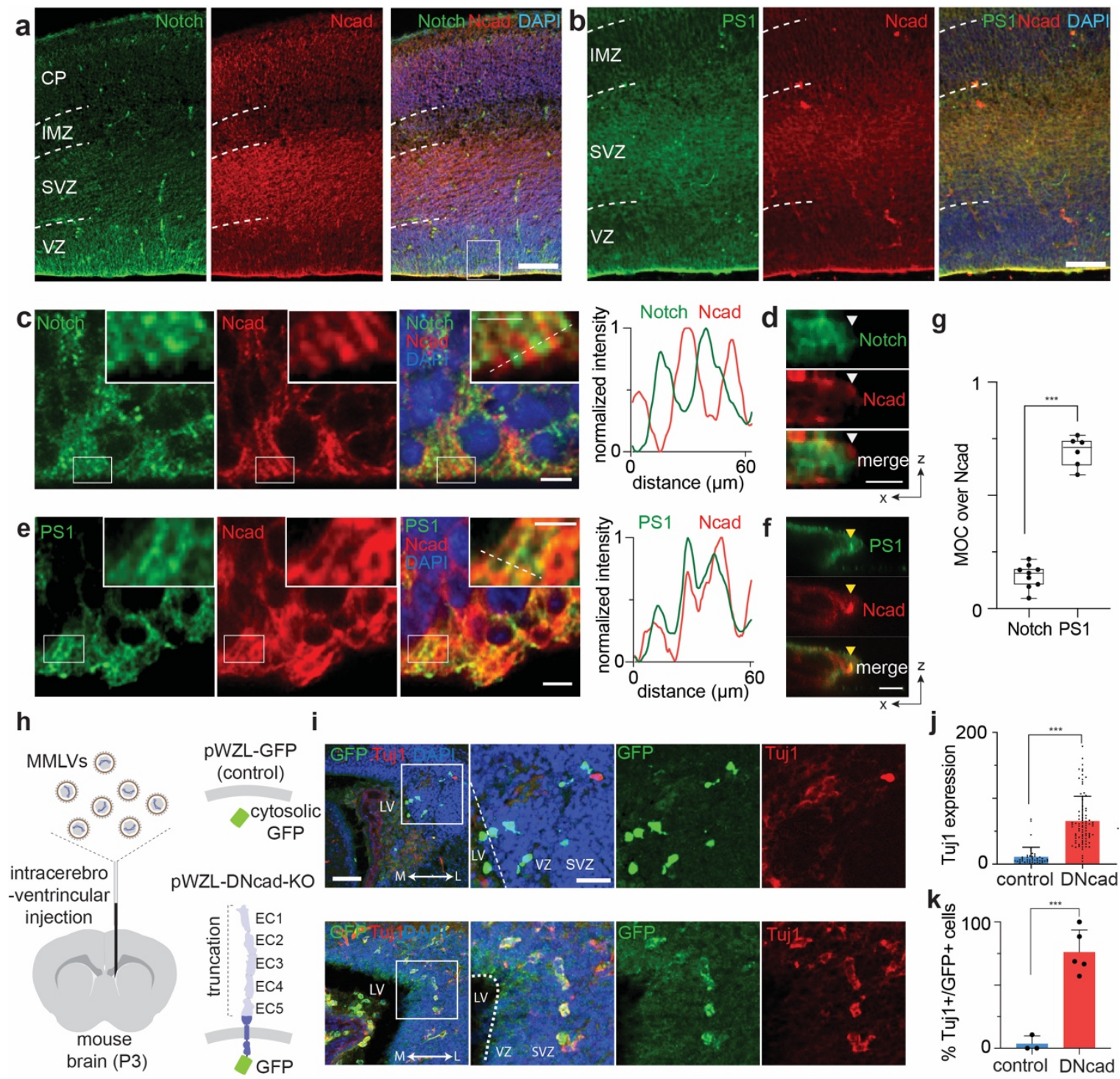

i
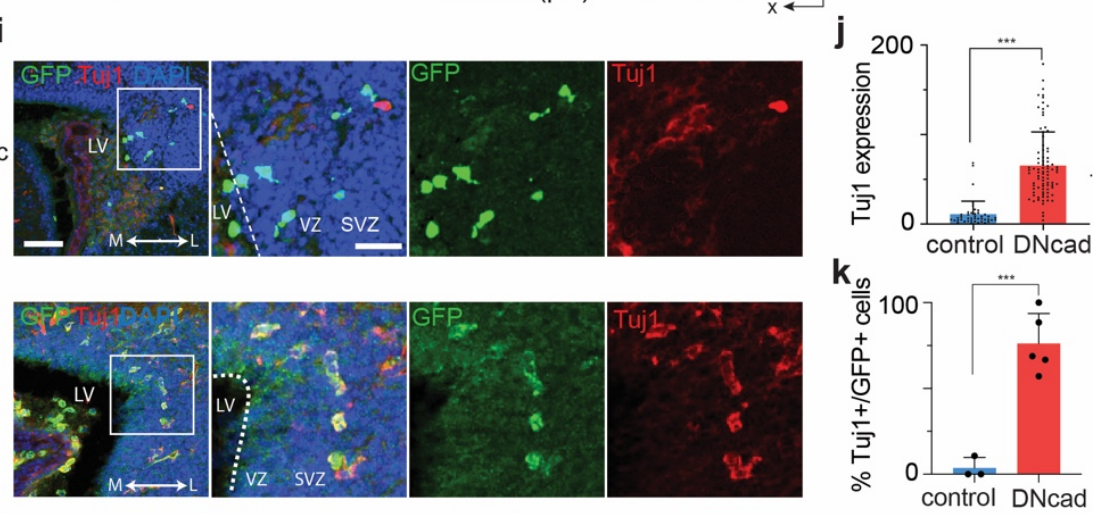

Fig. 6. The AJ-mediated spatial switch regulates neuronal progenitor cell differentiation in vivo. (a-f) Immunostaining of the subventricular zone (SVZ) in the lateral ventricle (LV) of the E13.5 mouse brain. Notch (a, c, d) and PS1 (b, e, f) distributions relative to AJs.) Representative low (a,b) and high (c,e) magnification images. Scale bars, $100 \mu \mathrm{m}$ and $5 \mu \mathrm{m}$, respectively. The boxed area in panels (c) and (e) is further magnified in the inset. Scale bar, $2.5 \mu \mathrm{m}$. Line profile analysis shown in panels (c) and (e). (d,f) Representative confocal z-resliced image showing Notch exclusion (white arrowhead) and PS1 colocalization (yellow arrowhead) with the AJ. Scale bar, $3 \mu \mathrm{m}$. (g) Quantitative assessment of Notch and PS1 colocalization with $\mathrm{N}$-cadherin in vivo. Each dot represents MOCs quantifying colocalized Notch (n $=9)$ or PS1 $(n=6)$ over selected AJs. (h) Retroviral infection of a plasmid encoding control vector (EGFP) or dominant negative form of E-cadherin vector (DN-cad-EGFP) to developing P3 mice via intracerebroventricular injection. (i) Retroviral infection of DN-cad-GFP increases the differentiation of NPCs compared with control. Cells differentiated into post-mitotic neurons can be identified as EGFP+/Tuj1+, while those which remained as NPCs with plasmid transfection are only EGFP+. Scale bar, $50 \mu \mathrm{m}$. (j) Quantification of the expression of Tuj1 per single cells ( $n=43$ cells across 3 mice and $n$ $=86$ cells across 5 mice per control and DN-cad, respectively). (k) Quantification of the percentage of Tuj1-expressing post-mitotic neurons among all transfected EGFP+ cells were quantified. Data are represented as mean \pm SEM. ${ }^{* *} p<0.01,{ }^{* * *} p<0.001$, two-tailed unpaired Student's t-test. 
a

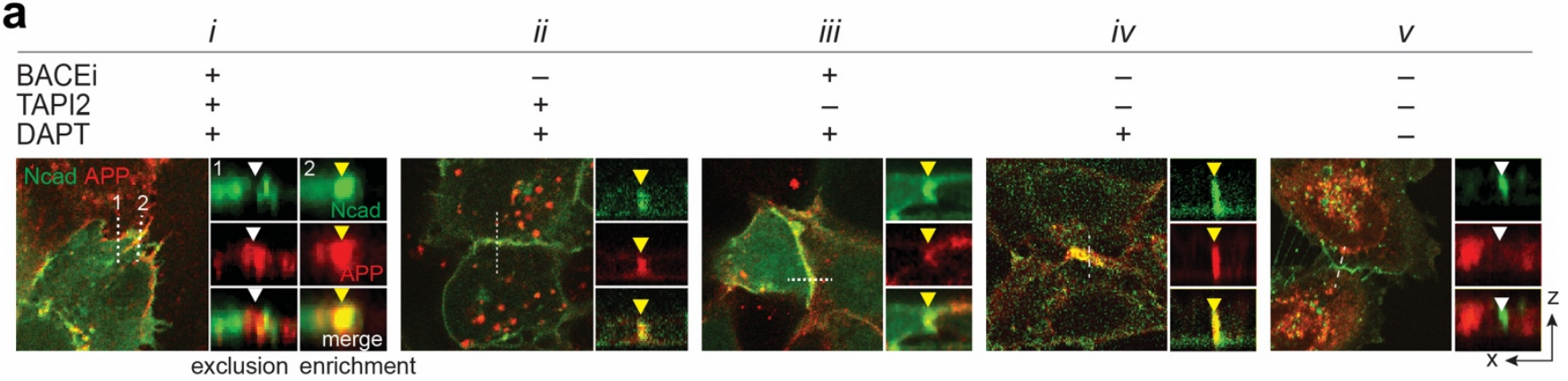
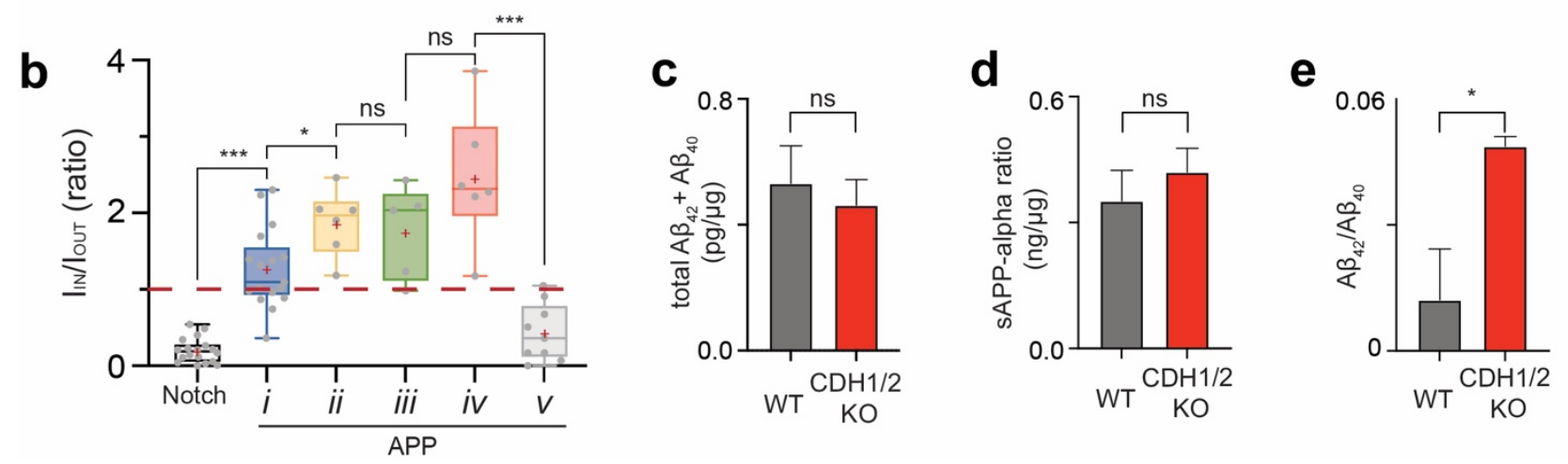

Fig. 7. The AJ-mediated spatial switch regulates APP signaling. (a) Confocal fluorescence maximum projection (left) and z-resliced images (right) of U2OS cells co-expressing Ncad-mCherry (green) and APP-EGFP (red) in different combinations of $\alpha$-, $\beta$-, and $\gamma$-secretase inhibitors. Scale bars, $10 \mu \mathrm{m}$ (maximum projection) and $3 \mu \mathrm{m}$ (inset). (b) Quantification of the enrichment factor ( $\mathrm{I}_{\mathrm{IN}} / \mathrm{l}_{\mathrm{OUT}}$ ) of APP signal relative to the $\mathrm{N}$-cadherin-based $\mathrm{AJs}$. Boxes and whiskers indicate the interquartile and full ranges, respectively. Black lines and $(+)$ marks indicate median and mean, respectively. ${ }^{*} \mathrm{P}<0.05 ;{ }^{* *} \mathrm{P}<0.01$; ${ }^{* * * * P}<0.001$; ns, not significant; ordinary one-way ANOVA followed by Tukey's multiple comparison. (ee) Total sum of $A \beta_{42}$ and $A \beta_{40}$ (c), soluble $A P P \alpha(\mathbf{d})$, and $A \beta_{42} / A \beta_{40}$ ratio (e) measured by ELISA in wildtype cells or $\mathrm{CDH} 1 / 2 \mathrm{KO}$ cells (mean $\pm \mathrm{SEM}, \mathrm{n}=3$, ${ }^{*} \mathrm{P}<0.05$, two-tailed paired Student's $\mathrm{t}$ test). 


\section{Online Methods}

\section{Plasmid construction}

Plasmid constructs used in this study are listed in Supplementary Table 1. All constructs used in this paper were assembled using standard restriction enzyme-based cloning, in-fusion cloning, and/or Gibson isothermal assembly. The maps, sequences, and construction details of all plasmids are available upon request. All constructs were sequenced to confirm mutation. Complete details of all cloning procedures are available upon request.

Flag-human Notch1 ( $\mathrm{N}^{\mathrm{FL}}$ )-Gal4 and pGF1-UAS-H2B-mCherry were gifts from S. Blacklow (Harvard University). Flag-human $\mathrm{N}^{\mathrm{FL}}$-Gal4 was provided in a Tet-ON Flp-IN vector (pcDNA5). SNAP-N ${ }^{\mathrm{FL}}$-mCherry and SNAP-N $\mathrm{N}^{\mathrm{FL}}$-Gal4 were constructed as previously reported ${ }^{38}$. All Notch1 variants with partial or full extracellular domain truncation were constructed by linearizing and amplifying SNAP-hN1-mCherry vector via inverse PCR while omitting the sequence corresponding the ECD truncation. Notch ectodomain sequences of amino acid 23-981, 23-1426, 23-1709 were deleted for SNAP- $\Delta \mathrm{EGF}_{1-25^{-}}$ mCherry. SNAP- $\triangle E G F-m C h e r r y$. and SNAP-NEXT-mCherry, respectively. Note that similar Notch variants with partial ECD truncation were reported previously ${ }^{78}$ where the structural integrity and function of Notch negative regulatory region (NRR) domain were preserved. Ecad-GFP was purchased from Addgene (Addgene plasmid \# 28009; http://n2t.net/addgene:28009). SNAP-Ecad-GFP and Halo-EcadGFP were constructed first by linearizing and amplifying Ecad-EGFP vector via inverse PCR. SNAP-and Halo-tags were then inserted in frame with E-cadherin, downstream of the E-cadherin pro-peptide (amino acid \#155) and upstream of the extracellular domain sequence, using Gibson assembly (NEB). pCMV6Flotillin-1-Halo (Flot1-Halo) was constructed by replacing the myc-tag within the pCMV6-Flotillin1-myc (purchased from Origene (MR206823)) with the Halo-Tag sequence in frame with Flot1 using In-Fusion cloning (Clontech). Human amyloid precursor protein1-EGFP (APP-EGFP) was created by cloning human APP695 (a gift from C. Miller of King's College London) into a pEGFP-N1 plasmid (Clontech). To facilitate membrane distribution mapping, we used APP constructs used in confocal imaging lack YENPTY motifs (Fig. 7a,b), and compared the result with full-length APP (Fig. 7a,b). APP ${ }^{\triangle Y_{E N P T Y}}$-EGFP was constructed by first linearizing the vector via inverse PCR, and deleting the sequence of final 15 amino acids upstream of C-terminus of APP (amino acid 681-695), which includes YENPTY motif (amino acid 682-687) using Gibson assembly.

\section{Tissue culture}

Human U2OS cells were cultured in McCoy's 5A media supplemented with $10 \%$ heat-inactivated FBS (Life Technologies) and 1\% Pen-Strep (Life Technologies) and passaged with $0.05 \%$ trypsin-EDTA. MDCK cells were grown in Eagle's Minimum Essential Medium (UCSF cell culture facility) supplemented with 10\% heat-inactivated FBS and 1\% Pen-Strep. MDCK cells were lifted by treating for 10 minutes with PBS $\mathrm{Ca}^{2+}$ and $\mathrm{Mg}^{2+}$ free with $0.05 \%$ EDTA followed by trypsinization. HUVECs were purchased from ATCC (ATCC $®$ CRL-1730 ${ }^{\mathrm{TM}}$ ) and grown in Endothelial Cell Growth Medium-2 (EGM-2, Lonza CC-3162) for up to 4 passages $(0.05 \%$ trypsin-EDTA) after thawing. Human immortalized keratinocyte cell line, $\mathrm{HaCaT}$, were purchased from ATCC (ATCC® PCS-200-011) and grown in DMEM (Gibson) supplemented with $10 \%$ FBS and $1 \%$ Pen-Strep. All cell lines were maintained at $37^{\circ} \mathrm{C}$ in a humidified incubator with $5 \% \mathrm{CO}_{2}$ and passaged every 2-3 days, depending on confluency, using $0.05 \%$ TrypsinEDTA (UCSF cell culture facility). For polarized MDCK culture, cells were for use on a transwell filter (0.4 $\mu \mathrm{m}$, collagen coated) at high density.

\section{$\underline{\text { Transfection and cell line generation }}$}

All cell lines expressing recombinant proteins used in this study are listed in Supplementary Table 1. U2OS stable cell lines were constructed from parental U2OS T-rex cell lines (FIp-IN, Tet-ON engineered cell line, gift from S. Blacklow). Constructs were inserted into the engineered Flp-IN site by co-transfection with a plasmid containing the Flp-recombinase (pOG44) via electroporation with the Neon Transfection System (ThermoFisher) according to manufacturer's protocol (Shock Conditions: 1230V, 10ms, 4 pulses, number of cells $5 \times 10^{6}$ ). The amount of total DNA used was $10 \mu \mathrm{g} /$ well: $1 \mu \mathrm{g}$ of DNA containing the 
desired construct and $9 \mu \mathrm{g}$ pOG44. Cells transfected with desired plasmids were incubated in a selection medium containing $400 \mu \mathrm{g} / \mathrm{mL}$ hygromycin (Invitrogen) for at least 10 days. All cells with Notch truncation and reporter were further sorted for inducible expression of Notch variants via fluorescence-activated cell sorting (FACS) on a FacsAria2 (BD) by staining for the appropriate tag (SNAP or Halo) with fluorescently tagged antibody. For single-cell monoclonal population establishment, fluorescently-positive bulk-sorted populations were plated into 96 well plates at 0.2 cells/well by serial dilution and grown in selection medium. Each clonal cell population was tested and selected based on the levels of Notch reporter activity or Notch membrane expression. U2OS cells expressing recombinant proteins transiently were generated by transfecting plasmids encoding desired proteins using Neon-based electroporation. Cells were allowed to settle in a 6-well cell culture dish post electroporation for 6-8 hr. To remove dead cells, cells were lifted and re-plated on a fibronectin-coated glass bottom dish with $1 \times 10^{5}$ cells per well density. MDCK cells were plated at $70 \%$ density then transfected with $\mathrm{N}^{\mathrm{FL}}$-mCherry utilizing Lipofectamine 3000 (ThermoFisher) or Neon electroporation according to the manufacturer's protocol (Shock Conditions: $1,650 \mathrm{~V}, 20 \mathrm{~ms}, 1$ pulse, number of cells $5 \times 10^{6}$ ). HUVEC cells were transfected via electroporation with SNAP-N ${ }^{\mathrm{FL}}$ via the BioRad Gene Pulser system ( $250 \mathrm{~V}, 20 \mathrm{~ms}$ square wave, $1 \times 10^{6} \mathrm{cells} / \mathrm{mL}$ Gene Pulser Electroporation Buffer, $5 \mu \mathrm{g} / \mathrm{mL}$ SNAP-N $\mathrm{NL}^{\mathrm{FL}} \mathrm{mC}$ ). All cells transiently expressing recombinant proteins were incubated for 24-48 hr from the transfection, and then used for further analyses.

\section{Live cell mechanogenetics experiment}

Mechanogenetics experiments were performed as previously described in Kwak et al., 2019 with some modifications ${ }^{38,53,54}$. Monovalent magnetofluorescent nanoparticles (MFNs) were synthesized as previously described ${ }^{54}$.

Micro-magnetic tweezers $(\mu M T)$ set up. The $\mu \mathrm{MT}$ was set up and aligned on the inverted microscope with point-scanning confocal imaging capabilities (Nikon) as previously described ${ }^{38,54,87}$. The needle probe NdFeB magnet assembly was attached to the z-translation stage (Sutter Instrument, MP-325) and its location was carefully aligned with the microscopic objective lens while observing the dummy substrate filled with DPBS. The $\mu \mathrm{MT}$ tip was positioned at the center of the objective oculus with bright-field illumination using the $X-Y$ translation stage linked to PIMikroMove (Physik Instrumente) and $\mu$ Manager (UCSF). Using the $z$ translation stage, the $\mu \mathrm{MT}$ was carefully lowered to set the height of the tip to $10 \mu \mathrm{m}$ above the focal plane while recording the $X-Y$ coordinates and the $Z$-position of the needle probe.

Preparation of cells expressing recombinant Flotilin-1 for mechanogenetics experiments. U2OS cells were co-transfected with SNAP-Ecad-GFP $(5 \mu \mathrm{g})$ and Flot1-Halo $(5 \mu \mathrm{g})$ plasmids using Neon electroporation. $24 \mathrm{hr}$ later, cells were re-plated on a \#1.5 glass-bottomed dish (MatTek, $d=10 \mathrm{~mm}$ ) coated with collagen at a density of $1 \times 10^{5}$ cells per dish. To fluorescently label Flot1-Halo, cells were treated in a complete McCoy's 5A medium containing $3.5 \mu \mathrm{M}$ cell membrane permeable Halo-ligand 660 dye (Promega) for 30 minutes at $37^{\circ} \mathrm{C}$. Cells were washed three times with DPBS, incubated with a phenol red-free complete medium, and then mechanogenetically stimulated (see below). For the cholesterol depletion experiment, we also treated cells $10 \mathrm{mM}$ of methyl- $\beta$-cyclodextrine (M $\beta C D$ ) (SigmaAldrich) in serum-free McCoy's $5 \mathrm{~A}$ medium for $30 \mathrm{~min}$ at $37^{\circ} \mathrm{C}$ and washed with complete medium three times. To label SNAP-Ecad-GFP with MFNs, cells were first treated with $5 \mu \mathrm{M}$ of an oligonucleotide bearing benzylguanine $\left(B G-T_{60} A C T G_{10}\right)$ for 45 minutes at $37^{\circ} \mathrm{C}$, washed two times with 10 ml of serum free medium, and then incubated with serum-free medium containing $10 \mathrm{nM}$ monovalent MFNs bearing complementary sequence $\left(\mathrm{T}_{60} \mathrm{CAGT}_{10}\right)$ and $0.5 \%$ alkali casein for $10 \mathrm{~min}$ at $37^{\circ} \mathrm{C}, 5 \% \mathrm{CO}_{2}$. Cells were washed with $10 \mathrm{ml}$ of complete medium two times, and then incubated with phenol red-free medium for mechanogenetic experiments on a confocal or wide-field epifluorescence microscope.

Preparation of cells expressing human Notch1 receptor for the mechanogenetic experiment. Inducible U2OS cells stably integrated with SNAP-N ${ }^{\mathrm{FL}}$-mCherry were transfected with Halo-Ecad-GFP $(10 \mu \mathrm{g})$ using Neon electroporation. $24 \mathrm{~h}$ later, cells were re-plated on a collagen (or fibronectin)-coated glassbottomed dish. To induce surface expression of SNAP-N $\mathrm{N}^{\mathrm{FL}}$-mCherry. cells were incubated with complete medium containing doxycycline (Sigma, $2 \mu \mathrm{g} / \mathrm{mL}$ ) for $18 \mathrm{~h}$. To inhibit $\gamma$-secretase activity, cells were treated with DAPT $(5 \mu \mathrm{M})$ and further incubated for $6 \mathrm{~h}$. Cells were treated with $5 \mu \mathrm{M}$ of an oligonucleotide 
bearing chloroalkane $\left(\mathrm{Cl}-\mathrm{T}_{60} \mathrm{ACTG}_{10}\right)$ for 45 minutes at $37^{\circ} \mathrm{C}$ and labeled with MFNs via the procedure described above.

Mechanogenetic regulation of artificial E-cadherin junctions. To induce MFN and hence cadherin clustering, the $\mu \mathrm{MT}$ was carefully directed towards a targeted subcellular location until the tip-tomembrane distance $(d)$ reached $10 \mu \mathrm{m}$. As the tip approached the target membrane, the formation of an artificial E-cadherin junctions (AJs) was monitored every 5 minutes. After 30 min of mechanogenetic stimulation, the spatial distribution of MFNs and artificial AJs was monitored using time-lapse confocal fluorescence imaging. To investigate $\gamma$-secretase processing of full-length Notch, the spatial distribution of membrane mCherry (S-N ${ }^{\mathrm{FL}}-\mathrm{mC}$ ) or nuclear mCherry signals (S-N $\left.{ }^{\mathrm{FL}}-\mathrm{Gal} 4\right)$ were monitored using timelapse live cell confocal imaging. To observe localization of membrane microdomains, the spatial distribution of Flot1 fluorescence signal was monitored using live cell confocal imaging. Time-lapse live cell confocal imaging was performed using a 60x Plan-Apo oil objective (NA 1.4) on a Nikon A1 laser scanning confocal microscope equipped with an environmental chamber maintaining cells at $37^{\circ} \mathrm{C}, 5 \%$ $\mathrm{CO}_{2}$. Cells were immediately fixed with $4 \%$ paraformaldehyde (Life Technologies) in DPBS for 15 minutes and washed with DPBS 3 times for 5 minutes before immunostaining.

\section{Fluorescence labeling and Immunostaining}

Fluorescence labeling of cells expressing SNAP- or Halo-tag proteins. Cells expressing SNAP- and/or Halo-tagged fusion proteins were labeled with BG- and/or chloroalkane functionalized fluorescence dyes, respectively. Dox-inducible cell lines grown on a collagen l-coated substrate were treated with doxycycline $(2 \mu \mathrm{g} / \mathrm{ml}) 24 \mathrm{hr}$ before labeling. Cells with transient receptor expression were labeled with dyes $48 \mathrm{hr}$ post-transfection. Dye-labeling was performed by treating the cells with $5 \mu \mathrm{M}$ fluorescence dye in serum containing media for $30 \mathrm{~min}$. Cells were then washed 3 times with complete media. For live cell imaging, cells were incubated with phenol-red free complete media. For imaging of fixed cells, cells were washed with PBS, fixed with 4\% PFA in PBS for 10 min, and then washed thoroughly with PBS.

Immunofluorescence staining. Fixed cells were permeabilized with $0.5 \%$ tween-20 diluted in PBS for 15 minutes, then blocked by incubation with blocking buffer ( $5 \%$ normal goat serum and $1 \%$ BSA in $1 \times P B S)$ for $1 \mathrm{hr}$ in room temperature. For immunostaining of surface ADAM10 and ADAM17 expression, the sample was directly blocked without a permeabilization step. Cells were incubated overnight at $4^{\circ} \mathrm{C}$ or 2 $\mathrm{hr}$ at $25^{\circ} \mathrm{C}$ with the primary antibodies: mouse monoclonal anti-ADAM10 (1:100; Santa Cruz Biotechnology), mouse monoclonal anti-ADAM17 (1:200, R\&D Systems), rabbit polyclonal anti-PS1 (1:50), rabbit monoclonal anti-Nicastrin (1:200, Santa Cruz Biotechnology), mouse polyclonal anti-Paxillin (1:500; BD Bioscience), rabbit polyclonal anti-myc tag (1:200; abcam), mouse monoclonal anti VEcadherin (1:400, BD Biosciences), and Alexa Fluor 488 Phalloidin (1:400 dilution of 200 units $/ \mathrm{mL}$ stock ; life technologies). All antibodies were diluted in the $0.5 \mathrm{x}$ blocking buffer $(2.5 \%$ normal goat serum and $0.5 \%$ BSA in 1xPBS). Following primary antibody incubation, cells were washed in PBS for 5 min four times and incubated with Alexa Fluor 405-conjugated goat anti-mouse (1:400, PS-1), Alexa Fluor 594conjugated goat anti-mouse (1:400; Paxillin), Alexa Fluor 488-conjugated goat anti-mouse (1:400; VEcadherin), and Alexa Fluor 647-conjugated goat anti-mouse (1:500, ADAM10), as appropriate. Nucleus staining was performed using Hoechst 33342 (ThermoFisher) diluted in 1xPBS $(1 \mu \mathrm{g} / \mathrm{mL}$ ) for 15 min and then washed once. Cells were incubated at room temperature in the dark for $1 \mathrm{hr}$, washed with PBS for 5 min three times, and imaged by epifluorescence or confocal microscopy. Epifluorescence microscopy was performed with 60x Apo, 1.40 NA or 100x Apo, 1.49 NA oil objectives (Nikon) on a Nikon Ti Eclipse microscope equipped with OBIS solid-state lasers (488, 552, and $647 \mathrm{~nm}$, Coherent Inc.), a $300 \mathrm{~W}$ Xenon lamp (Sutter Instrument, Lambda LS), a motorized stage (ASI, MS-2000), and a temperature- and $\mathrm{CO}_{2-}$ controlled stage top incubator (Okolab, Bold Line). Unless otherwise noted, confocal microscopy was performed using Plan-Apo 60x, 1.4 NA or Plan-Apo 100x, 1.4 NA oil objectives (Nikon) on a Nikon A1R laser scanning confocal microscope. Images were acquired using Galvano scanning mode and confocal zoom of 3-4x magnification.

Monitoring the dynamic spatial localization of Notch intermediates 
To activate Notch, we plated cells expressing SNAP-N ${ }^{\mathrm{FL}}-\mathrm{mCherry}$ on a substrate coated with DII4 fused with a Fc fragment (DII4-Fc). Briefly, a glass bottom dish (Lab-Tek II Chambered Coverglass, ThermoFisher, or 7-mm glass-bottomed dish, MatTek) was coated with fibronectin (Hamster, $5 \mu \mathrm{g} / \mathrm{ml}$ ) and DIl4-Fc $(2.5 \mu \mathrm{g} / \mathrm{ml})$ for $1 \mathrm{hr}$ at $37^{\circ} \mathrm{C}$, and washed thoroughly with $10 \mathrm{ml}$ of PBS. A negative control dish was also prepared by coating it with fibronectin only. U2OS cells co-expressing SNAP-N ${ }^{\mathrm{FL}}$-mCherry and Ecad-GFP were plated and incubated with doxycycline $(2 \mu \mathrm{g} / \mathrm{mL})$, TAPI2 $(100 \mu \mathrm{M}){ }^{88}$, and/or DAPT $(5 \mu \mathrm{M})$. Different combinations of inhibitors were used to capture the respective intermediates (See Fig. 1a-d and Extended Data Fig. 2a-c). After 48 hr, cells were labeled with SNAP-647 (NEB, $5 \mu M$ ) and then fixed as detailed above. Inhibitor concentrations were maintained during wash and fixation steps. For DAPT washout experiments cells were plated and activated via DII4-Fc ligand as described above. DAPT inhibitor was removed by washing in media at each time point $(0,0.5,1.5,3$, 6 , and $12 \mathrm{hrs})$. At each time point, cells were washed three times with large volumes of PBS and fixed $4 \%$ with PFA. The spatial distribution of Notch intermediates and AJs was monitored using spinning disk confocal fluorescence microscopy (Zeiss Cell Observer Z1), equipped with Yokagawa spinning disk and Evolve 512 EMCCD Camera (Photometrics). Images were obtained with Plan-Apo 63x, 1.4 NA or Plan-Apo 100x 1.46 NA oil objectives (Zeiss) with solid-state lasers of 405, 488, $561 \mathrm{~nm}$, and $647 \mathrm{~nm}$. The microscope was controlled with Zeiss Zen software (Zeiss).

\section{Plasma membrane staining using Dil dyes}

U2OS cells expressing Ecad-GFP were plated on a fibronectin-coated glass bottom dish (MatTek, D = $7.0 \mathrm{~mm}$ ) at a density of $1 \times 10^{4}$ cells per dish. After $48 \mathrm{hr}$, the dish was filled with a complete McCoy's 5A medium containing $5 \mu \mathrm{M}$ of Dil plasma membrane labeling dyes (Invitrogen) and incubated for 10 min at $37^{\circ} \mathrm{C}, 5 \% \mathrm{CO}_{2}$. Cells were then washed 3 times with complete medium. The spatial distribution of AJs and Dil membrane staining and Ecad-GFP was monitored using spinning disk confocal microscopy (See Extended Data Fig. 3f).

\section{Single-cell cleavage kinetics of SNAP-N $\triangle E$ EF-mC}

A 6-channel $\mu$-slide flow chamber (Ibidi, VI 0.4) was coated with fibronectin $(2.5 \mu \mathrm{g} / \mathrm{mL})$ for $1 \mathrm{hr}$ at $37^{\circ} \mathrm{C}$ and washed with PBS four times. U2OS cells co-expressing SNAP-N $\Delta E G F-m C h e r r y$ and Ecad-GFP were plated on the $\mu$-slide flow chamber by applying $60 \mu \mathrm{L}$ of single cell suspension at a density of $3 \times$ $10^{5}$ cells $/ \mathrm{mL}$. After $3 \mathrm{hr}$, the channel was filled with a complete McCoy's $5 \mathrm{~A}$ medium containing doxycycline $(2 \mu \mathrm{g} / \mathrm{mL})$, TAPI2 $(100 \mu \mathrm{M})$, and DAPT $(5 \mu \mathrm{M})$. Cells were grown for $48 \mathrm{hr}$ in normal growth medium to reach $70-80 \%$ confluency and form cadherin adherens junctions. Cells were labeled with BGAlexa Fluor 647 (NEB) for 30 min to stain cell surface N $\Delta$ EGF. Multiple cells with stable AJs were identified using large-area epi-fluorescence scanning $(500 \mu \mathrm{m} \times 500 \mu \mathrm{m})$, and the spatial distribution of SNAP-N $\triangle E$ EGF-mCherry at AJs under TAPI2 and DAPT inhibition was imaged by confocal z-stack (step size $=0.2 \mu \mathrm{m}$, total range of $z$ stacks $=10 \mu \mathrm{m}$ ) scanning from basal to apical membranes. Then, DAPT containing media was removed and replaced by flowing complete medium containing doxycycline and TAPI2 at a flow rate of $50 \mu \mathrm{l} / \mathrm{min}$ for 10 minutes using a syringe pump. Localization of extracellular (NECD) and intracellular (NICD) domain at the AJs before and during DAPT washout was monitored every 30 minutes in multiple color channels (NICD, mCherry; NECD, AF647; AJ, GFP) by time-lapse confocal zstack microscopy for $12 \mathrm{hr}$. Time-lapse live cell confocal imaging was performed using a 60x Plan-Apo oil objective (NA 1.4) on a Nikon A1 laser scanning confocal microscope equipped with an environmental chamber maintaining cells at $37^{\circ} \mathrm{C}, 5 \% \mathrm{CO}_{2}$.

\section{Western Blot analysis}

U2OS cells co-expressing Notch variants and Ecad-GFP (or Halo-Ecad-GFP) were incubated with culture media containing doxycycline $(2 \mu \mathrm{g} / \mathrm{mL})$ and TAPI2 $(100 \mu \mathrm{M})$ in a 6 -well plate at a density of $1 \times 10^{6}$ cells per well. After $24 \mathrm{hr}$, cells were washed with ice-cold DPBS twice and lysed in RIPA (Invitrogen) or 1\% NP-40 (Invitrogen) supplemented with complete protease and phosphatase inhibitor cocktail (100x; Cell Signaling Technology) at $4^{\circ} \mathrm{C}$ while gently shaking for 30 minutes. Insoluble fractions were removed by 
centrifugation of the cell lysates at 13,000 r.p.m. for 10 minutes. Total protein concentrations in lysates were determined by a BCA assay (Bio-Rad). $20 \mu \mathrm{g}$ of whole cell lysates were then mixed with $4 x$ Laemmli sample buffer (Bio-Rad) with 10\% $\beta$-mercaptoethanol (BME) and heated to $95^{\circ} \mathrm{C}$ for 5 minutes. For western blot analysis of DNA-crosslinked heterodimers, the cell lysates were mixed with 4x Laemmli sample buffer (Bio-Rad) without BME before boiling to denature. Samples were then loaded into a 4-15\% Mini-Protein TGX precast gel (Bio-Rad) and were run at $70 \mathrm{~V}$ for 30 minutes and then $120 \mathrm{~V}$ for 45 minutes. Separated proteins were transferred to a PVDF membrane using Mini Trans-Blot Cell (100 V constant, $1 \mathrm{hr}$ ) or the Trans Turbo Blot system (Bio-Rad). Membranes were blocked for $1 \mathrm{hr}$ at room temperature in blocking solution ( $5 \% \mathrm{w} / \mathrm{v}$ nonfat dry milk in 1x TBST). The membranes were probed with anti-V1744 NICD antibody (1:1000; Cell Signaling Technology \#4147), anti-SNAP (1:1000; NEB), antiNotch1 (1:1000, Cell Signaling Technology \#3447 or \#4380), anti-mCherry (1:500, Abcam \#167453), anti-E-cadherin (1:100, Santa Cruz Biotechnology, sc-8426), and anti- $\beta$-actin (1:5000; Cell Signaling Technology \#4970) antibodies overnight at $4^{\circ} \mathrm{C}$ with gentle rocking. The membranes were washed in TBST three times for 5 minutes and incubated with an anti-rabbit (Cell Signaling Technology, \# at 1:2000 for NICD, mCherry, SNAP detection and at 1:10000 for $\beta$-actin detection) or anti-mouse (Cell Signaling Technology, \# at 1:2000 for E-cadherin detection) HRP conjugated antibody. The target proteins were visualized by chemiluminescence using an ECL detection kit and a ChemiDoc MP imaging system (BioRad). Quantification of band intensities by densitometry was carried out using the Image Lab software (Bio-Rad).

\section{Spatial mutation of SNAP-N $\triangle E$ EF-mCherry via DNA crosslinking}

DNA-mediated crosslinking of SNAP-N $A E G F-m$ Cherry with Halo-Ecad-GFP. DNA crosslinkers including benzylguanine (BG)- and chloroalkane $(\mathrm{Cl})$ modified oligonucleotides were synthesized as previously described (Kwak et al., 2019; Liang et al., 2018). To prepare 10x crosslinking DNA stock solution, complementary BG- and Cl-modified oligonucleotides were hybridized in situ. BG-T10(ACTG $)_{5}$ and $\mathrm{Cl}-$ $\mathrm{T}_{10}(\mathrm{CAGT})_{5}$ were mixed at equimolar concentration $(20 \mu \mathrm{M})$ in $\mathrm{PBS}$, incubated at $95^{\circ} \mathrm{C}$ on a dry heat block for $5 \mathrm{~min}$, and slowly cooled down to room temperature for $2 \mathrm{hr}$. U2OS cells co-expressing SNAP$\mathrm{N} \triangle \mathrm{EGF}$-mCherry and Halo-Ecad-GFP were cultured in a 6-well plate for western blot analysis at a density of $1 \times 10^{6}$ cells per $\mathrm{mL}$ or in a channel of an Ibidi $\mu$-slide for confocal imaging analysis at a density of $3 \times$ $10^{5}$ cells per $\mathrm{mL}$. Cells were grown to $70-80 \%$ confluency for typically $24 \mathrm{hr}$, followed by overnight incubation with complete medium containing doxycycline $(2 \mu \mathrm{g} / \mathrm{mL})$, TAPI2 $(100 \mu \mathrm{M})$ and DAPT $(5 \mu \mathrm{M})$. Cells were then serum starved with $2 \mathrm{ml}$ of serum-free medium with doxycycline, DAPT, and TAPI2 for 6 hrs. Before adding DNA crosslinkers, cells were washed and placed in $450 \mu \mathrm{l}$ of serum-free media. $50 \mu \mathrm{l}$ of prewarmed 10x DNA crosslinker stock solution was added to each well and incubated at $37^{\circ} \mathrm{C}$. Western blot analysis to validate receptor crosslinking were performed after 30-minute incubation of the DNA crosslinkers as detailed above.

Live cell confocal time-lapse imaging. After overnight incubation with the DNA crosslinkers, imaging was performed on an inverted laser scanning confocal microscope (Nikon A1) equipped with an environmental chamber at $37^{\circ} \mathrm{C}$ and $5 \% \mathrm{CO}_{2}$. Images were obtained with a Plan-Apochromat $60 \mathrm{x}, 1.4 \mathrm{NA}$ oil objective (Nikon) with solid-state lasers of 405, 488, $561 \mathrm{~nm}$, and $647 \mathrm{~nm}$. Additionally, the microscope was equipped with Ti-E Perfect Focus System (Nikon). To examine the effect of DNA-mediated crosslinking on spatial distribution of SNAP-N $\triangle E$ EGF-mCherry at AJs, multiple AJs were imaged in entirety from basal to apical sides for Halo-Ecad-GFP and SNAP-N $\triangle E$ EGF-mCherry using a $488 \mathrm{~nm}$ and $561 \mathrm{~nm}$ laser respectively, for a $12 \mu \mathrm{m}$ range at a z-step size of $0.25 \mu \mathrm{m}$. To monitor dissipation of SNAP-N $\Delta E G F-$ mCherry at AJs upon removal of DAPT inhibition, fresh phenol red-free McCoy's 5A medium containing doxycycline and TAPI2 was introduced into the channel using a syringe pump for 10 min, and confocal z-stack images of the previously selected AJs were acquired every 30 minutes for $6 \mathrm{hr}$. Images were acquired using NIS-element software (Nikon), and image post-processing and analyses were done using Fiji/lmageJ and custom-built scripts.

Spatial mutation of SNAP-NEXT-mCherry via molecular pendant addition 
Synthesis of BG-modified polyethylene glycol (PEG). Amine-functionalized PEGs with different molecular weights and structures were purchased from Creative PEGWorks $\left(\mathrm{NH}_{2}-\mathrm{PEG} 3.4 \mathrm{k}\right)$, Sigma $\left(\mathrm{NH}_{2}-\right.$ $b$ PEG20k), and NanoCS ( $\mathrm{NH}_{2}-\ell P E G 20 \mathrm{k}$ ) and used without further purification. BG-functionalization of PEGs was performed by amine-NHS (N-hydroxysuccinimide; NEB) coupling reaction. Briefly, $\mathrm{NH}_{2}-\mathrm{PEG}$ $(0.5 \mu \mathrm{mol})$, BG-GLH-NHS $(2.4 \mathrm{mg}, 5 \mu \mathrm{mol})$, and N,N-dimethylaminopyridine $(0.73 \mathrm{mg}, 6 \mu \mathrm{mol}$; Sigma) were dissolved in anhydrous dimethylsulfoxide (DMSO). The mixture allowed to react overnight with constant shaking. Crude products were recovered by evaporating DMSO using a Speed-Vac concentrator (Vacufuge, Eppendorf), reconstituted in $500 \mu \mathrm{l}$ deionized water, and insoluble precipitates were removed by centrifugation at 14,000 r.p.m. for 10 minutes. The BG-modified PEG was then dissolved in $200 \mu \mathrm{l}$ in deionized water and purified by reverse-phase high performance liquid chromatography with an Agilent Eclipse XDB C-18, $5 \mu \mathrm{m}, 4.6 \times 250 \mathrm{~mm}^{2}$ column using an elution gradient of $5-75 \%$ acetonitrile in $0.02 \%$ trifluoroacetic acid.

Synthesis of BG-modified DNA-streptavidin conjugates. DNA oligonucleotides bearing biotin- and BGfunctional groups were synthesized by reacting biotin-(ACTG) ${ }_{5}-\mathrm{NH}_{2}$ (IDT DNA) with BG-GLH-NHS as described above ${ }^{63}$. Equimolar amounts of streptavidin $(10 \mathrm{nmol})$ and BG-DNA-biotin $(10 \mathrm{nmol})$ were dissolved in PBS $(0.5 \mathrm{ml})$ for $2 \mathrm{hr}$, forming streptavidin-BG complex. The solution was concentrated to approximately $50 \mu \mathrm{l}$ using an Amicon centrifugal filter (MWCO: 30k) and then diluted again with $0.45 \mathrm{ml}$ of PBS. This concentration and reconstitution step was repeated three times to remove unconjugated DNA.

Synthesis of BG-modified human IgG. hlgG (10 mg) and BG-GLA-NHS (0.82 mg) were dissolved in 850 $\mu \mathrm{l}$ of PBS and $150 \mu \mathrm{l}$ of anhydrous DMSO, respectively. Two solutions were mixed and reacted for $2 \mathrm{hr}$ at room temperature with gentle shaking. The solution was desalted with NAP-10 and then with NAP25 pre-equilibrated with PBS. The proteins were further concentrated until the final volume is $300-500 \mu \mathrm{l}$ using Amicon centrifugal filter (MWCO: 30k). The IgG concentration was determined by measuring the absorbance at $280 \mathrm{~nm}$.

Spatial mutation of SNAP-NEXT-mCherry using the BG-modified macromolecules. U2OS cells coexpressing SNAP-NEXT-mCherry and Ecad-GFP were incubated in complete McCoy's 5A medium containing doxycycline $(2 \mu \mathrm{g} / \mathrm{ml})$, TAPI2 $(100 \mu \mathrm{M})$, DAPT $(5 \mu \mathrm{M})$, and respective BG-modified macromolecules $(10 \mu \mathrm{M})$. After $24 \mathrm{~h}$, cells were fixed and imaged by confocal microscopy to determine the enrichment factor of SNAP-NEXT-mCherry at AJs. Images were taken with a 100x objective and 3x confocal zoom. 20 stage positions per each treatment were manually selected and their coordinates were stored in the computer. In each position, confocal z stacks of DAPI, Ecad-GFP and Notch-mCherry were acquired for a $12 \mu \mathrm{m}$ range at a $Z$ step-size of $0.25 \mu \mathrm{m}$ to monitor the AJs in their entirety from basal to apical sides. To assess the levels of Notch activation, a set of identical experiment but without DAPT was performed. After $24 \mathrm{~h}$, cells were fixed, stained with DAPI, and imaged by confocal microscopy to determine nuclear mCherry signal. Images were taken with a $60 x$ objective and 1x confocal zoom. 5 stage positions per each condition were selected manually. For each position, a confocal large-area scan of DAPI, Ecad-GFP, and SNAP-NEXT-mCherry was acquired for a $1 \mathrm{~mm} \times 1 \mathrm{~mm}$ area.

\section{Plate-bound DII4 Notch activation in high-density grouped versus solitary cells}

To activate Notch, we plated SNAP-N ${ }^{\mathrm{FL}}$-Gal4 reporter cells on a substrate coated with DII4-Fc as detailed above. Two different cell seeding densities were used: We plated cells with a density of $1 \times 10^{3}$ cells per $10 \mathrm{~mm}$ glass-bottomed dish (MatTek, No. 1.5 glass), predominantly yielding solitary cells. We also plated cells with a density of $1 \times 10^{4}$ cells per dish, predominantly yielding high-density grouped cells.

Plate-bound E-cadherin Notch activation experiment. Glass-bottomed dishes (MatTek, \#1.5, D = $10 \mathrm{~mm}$ ) were coated with recombinant human E-cadherin-Fc (50 $\mathrm{gg} / \mathrm{ml}$, R\&D systems), recombinant human DII4Fc $(2.5 \mu \mathrm{g} / \mathrm{ml}$, Sino Biological $)$, and fibronectin $\left(5 \mu \mathrm{g} / \mathrm{ml}\right.$, Sino Biological) diluted in PBS for $1 \mathrm{hr}$ at $37^{\circ} \mathrm{C}$, and rinsed with $10 \mathrm{ml}$ PBS with calcium and magnesium (UCSF cell culture facility). The U2OS SNAP$\mathrm{N}^{\mathrm{FL}}$-Gal4 reporter cells were transfected with Ecad-GFP $(10 \mu \mathrm{g})$ via electroporation, incubated overnight, and re-plated onto a fibronectin, E-cadherin-Fc, and Dll4-Fc coated glass-bottomed dish at a density of $0.3 \times 10^{5} \mathrm{cells} / \mathrm{ml}$, same as the solitary cell assay. Negative control experiment was performed with the cells plated on E-cadherin-Fc and fibronectin coated glass-bottomed dishes without DII4-Fc coating. 
Time-lapse epifluorescence imaging. All cells were treated with $2 \mu \mathrm{g} / \mathrm{ml}$ doxycycline (sigma-aldrich) at the time of plating. $2 \mathrm{hr}$ post-plating, cells were imaged using time-lapse microscopy. For a high-density cell seeding assay, several groups of cells having cell-cell contacts were manually identified and their coordinates were stored. For a solitary cell assay, a number of solitary cells without any prior cell-cell contact were manually identified and their coordinates were stored. While maintaining live cells on a microscope stage with a top stage incubator, time-lapse fluorescence images were acquired in GFP and mCherry channels. In each position, the microscope (Nikon) first found focuses using the Perfect Focus System (Nikon), took a DIC image, and two fluorescent images (GFP, mCherry). To image multiple solitary cells and grouped cells in one large image, cells were first plated at a high-density $\left(2 \times 10^{5} / \mathrm{ml}\right)$ at the center, and after $15 \mathrm{~min}$, cells were seeded at a low-density $\left(2 \times 10^{3} / \mathrm{ml}\right)$ over the entire substrate area. After $24 \mathrm{~h}$, cells were fixed and stained for membrane and nucleus. Epifluorescence images were obtained with an inverted microscope (Nikon, Ti Eclipse) equipped with 300W Xenon lamp (Sutter Instrument, Lambda LS), a motorized stage (ASI, MS-2000), and a temperature- and $\mathrm{CO}_{2}$-controlled stage top incubator (Okolab, Bold Line). Images were taken with 40x (CFI Plan fluor, N.A. 1.3, Nikon) objective lens. The microscopy setup was controlled using $\mu$-manager software.

\section{$\underline{\text { CRISPR editing to generate E-cadherin and N-cadherin knockout mutants }}$}

CRISPR/Cas 9 was used to knock out E-cadherin and N-cadherin expression from U2OS SNAP-N ${ }^{\mathrm{FL}}$-Gal4 reporter cells. The genes coding for the E-cadherin $(\mathrm{CDH} 1)$ and $\mathrm{N}$-cadherin $(\mathrm{CDH} 2)$ protein from homo sapiens (gene ID: ENSG00000039068 and ENSG00000170558) were truncated by a CRISPR/Cas9 paired sgRNAs excision strategy ${ }^{89,90}$. For the fragment deletion of genomic DNA, we used a pair of gRNAs against the target locus of CDH1 (Exon $1 \& 2$ (940bp deletion) or 13 \& 14 ( 4712bp deletion) (Extended Data Fig. 5a) and CDH2 (Exon $1 \& 2$ ( 29,255bp deletion) (Extended Data Fig. 5b) genes.

sgRNA design and expression vector cloning. Cas9 and sgRNAs were expressed using the CMV promoter-driven Cas9-2A-mRFP-2A-Puro plasmid (hereafter, Cas9-puro vector) and the hU6 promoterdriven sgRNA plasmid (Toolgen), respectively. To design sgRNAs for fragmental deletion of target loci of genes, all candidate sgRNA target sites with a protospacer adjacent motif (PAM; 5'-NGG-3') within the coding sequence of $\mathrm{CDH} 1$ and $\mathrm{CDH} 2$ were initially identified. For efficient deletion, selected sgRNAs for the candidate target sites were evaluated with DeepSpCas9 sgRNA prediction tool (http://deepcrispr.info/DeepSpCas9/) ${ }^{91}$. sgRNAs with high DeepSpCas9 score were selected and sgRNA oligonucleotides annealed and cloned into the vector as previously described (Ramakrishna et al., 2014a). Sequences of the vectors and sgRNAs listed here are available upon request.

Generation of single-cell derived knock-out clones. For CDH1 knockout, SNAP-N ${ }^{\mathrm{FL}}$-Gal4 reporter cells were transfected with plasmid mixtures containing Cas9-puro, U6-sgRNA encoding individual sgRNAs at a weight ratio of $1: 2$ using the Neon system. For $\mathrm{CDH} 1 / 2$ knockout, cells were transfected with plasmid mixtures containing Cas9-puro, U6-sgRNA targeting CDH1 loci, and U6-sgRNA targeting CDH2 loci at a weight ratio of 1:1:1 using the Neon system. One day after transfection, puromycin was added to the culture media at a final concentration of $2.5 \mu \mathrm{g} \mathrm{ml}^{-1}$. Three days after transfection, the pooled cells were analyzed for the indel efficiency of sgRNA pairs using T7E1 assay. To obtain single cell-derived clones containing the fragment deletion, we plated the cells after puromycin selection into 96-well plates at an average density of 0.25 cells/well. 14 days after plating, individual clones were isolated and analyzed using PCR and gel electrophoresis of genomic DNA to check the deletion and wild-type alleles as previously described (Ramakrishna et al., 2014b). We next sequenced the genomic DNA of the clones containing targeted deletions to check if the two cleavage sites were joined by the generation of indels. Sequencing of genomic regions including the target sequence was performed as previously described ${ }^{94}$. Briefly, PCR amplicons that included the junction regions of the deleted IncRNA target sites were cloned into the T-Blunt vector (Promega) and sequenced using universal M13FP or RP primers.

T7E1 assay. The T7E1 assay was performed as previously described ${ }^{95}$. Briefly, genomic DNA was isolated using the Wizard Genomic DNA purification Kit (Promega) according to the manufacturer's instructions. The region including the target site was nested PCR-amplified using appropriate primers. The amplicons were denatured by heating and annealed to allow the formation of heteroduplex DNA, which was treated with 5 units of $\mathrm{T} 7$ endonuclease 1 (NEB) for $20 \mathrm{~min}$ at $37^{\circ} \mathrm{C}$ followed by analysis using 
$2 \%$ agarose gel electrophoresis. Mutation frequencies were calculated as previously described based on the band intensities using Image J software and the following equation ${ }^{95}$ : mutation frequency $(\%)=100 \times$ $\left(1-(1-\text { fraction cleaved })^{1 / 2}\right)$, where the fraction cleaved is the total relative density of the cleavage bands divided by the sum of the relative density of the cleavage bands and uncut bands.

$R T-P C R$. Total RNA was extracted from wild-type SNAP-N ${ }^{\mathrm{FL}}$-Gal4 reporter (WT) cells and knockout clonal cells using TRIzol (Ambion) or an RNeasy Kit (QIAGEN), after which complementary DNA (cDNA) synthesis was performed using a DiaStarTM RT Kit (SolGent Co., Ltd.). The synthesized cDNA was subjected to quantitative PCR (qPCR) in triplicate using an Applied Biosystems StepOnePlus Real Time PCR System with PowerSYBR Green PCR Master Mix (Applied Biosystems). Gene expression was normalized to that of the CDH1 gene in WT cells. Error bars represent the standard deviation (s.d.) of the mean of triplicate reactions. Primer sequences for qPCR are available upon request.

Notch activation assay. WT cells, CRISPR CDH1 knock-out cells $\left(\mathrm{CDH} 1^{-/}\right), \mathrm{CDH} 1^{-/}$transfected with Ecadherin-GFP $\left(\mathrm{CDH}^{-/-}+\mathrm{E}\right.$-cad), and $\mathrm{CDH} 1^{-/-}$transfected with $\mathrm{N}$-cadherin $\left(\mathrm{CDH} 1^{-/-}+\mathrm{N}\right.$-cad) were plated on 8 well Nunc Lab-Tek II chambered coverglass pre-coated with recombinant human DII4-Fc $(2.5 \mu \mathrm{g} / \mathrm{ml})$ and fibronectin $(5.0 \mu \mathrm{g} / \mathrm{ml})$ as previously described. All cells were plated at a density of 30,000 cells per well. After $24 \mathrm{hr}$ incubation with doxycycline $(2 \mu \mathrm{g} / \mathrm{ml})$, cell cytoplasm and nucleus were stained with CellTracker CMFDA dye (Invitrogen) and Hoechst 33342 (ThermoFisher), respectively. The cells were then fixed with $4 \%$ PFA for 15 minutes at room temperature and proceeded to epifluorescence and confocal imaging.

\section{$\underline{\mathrm{A} \beta 40, \mathrm{~A} \beta 42, \text { and SAPP } \alpha \text { ELISA }}$}

$1 \times 10^{6}$ of wild-type or $\mathrm{CDH} 1 / \mathrm{CDH} 2$ knockout cells were transfected with APP-mCherry $(10 \mu \mathrm{g})$ and plated on 6-well tissue culture plate. After $48 \mathrm{hr}$ incubation, conditioned media supplemented with $1 \mathrm{x}$ protease/phosphatase inhibitor cocktail (ThermoFisher) were centrifuged at $3,000 \times \mathrm{xg} 10$ minutes at $4^{\circ} \mathrm{C}$ to remove cell debris and the supernatant were transferred to a new tube and stored at $-80 \mathrm{C}$. The conditioned media were analyzed for $A \beta 40, A \beta 42$, and sAPP $\alpha$ contents using A $\beta$ (Invitrogen) and sAPP $\alpha$ (Kusa Biosci.) ELISA kits. All ELISAs were performed according to the manufacturer's protocols. Briefly, $50 \mu \mathrm{l} /$ well of samples, followed by $50 \mu \mathrm{l} /$ well of detection antibodies, were applied to A $\beta 40, A \beta 42, \operatorname{sAPP} \alpha$ coated 96-well plate, and incubated for $3 \mathrm{hr}$ at room temp with shaking at $300 \mathrm{rpm}$. After washing step, $100 \mu \mathrm{l}$ of HRP-IgG solution was applied and incubated for $30 \mathrm{~min}$ at room temp with shaking. After washing step, $100 \mu \mathrm{l}$ of stabilized substrate solution was applied and incubated for 30 min at room temp with shaking. Finally, $100 \mu \mathrm{l}$ of stop solution was applied. Absorbance at $450 \mathrm{~nm}$ was read and analyzed using a plate reader (Biotek Synergy 2). Wells were washed with wash buffer 4 times between each incubation step. Standard curves were generated using recombinant $A \beta 40, A \beta 42$, sAPP $\alpha$ provided by the manufacturer (Invitrogen).

\section{$\underline{\text { In vivo experiments }}$}

Animals. We used CD-1 embryonic day 13.5 (E13.5) and postnatal day three (P3) newly born mice for in vivo experiments. P3 pups were obtained by purchased of an untimed pregnant female mouse (E13-15) from Charles River Laboratories (Wilmington, MA) and waited for birth. All mice were housed under specific pathogen-free conditions under a $12 \mathrm{~h}$ light-dark cycle, and all animal handling and use were in accordance with institutional guidelines approved by the University of California San Francisco Institutional Animal Care and Use Committee (IACUC).

Retrovirus injection. To generate retrovirus, we used pWZL-GFP control vector (pWZL-Blast-GFP: addgene plasmid \# 12269) and pWZL-dominant negative E-cadherin (pWZL-Blast-DN-E-cadherin addgene plasmid \# 18800 and gift from Dr. Kenji Shimamura). GFP sequence was inserted in frame with dominant negative E-cadherin, downstream of C-terminus using In-Fusion cloning. For retrovirus production, we transfected retroviral vectors into Phoenix-Ampho cells using Calcium Phosphate transfection kit (Sigma, CAPHOS) with $50 \mu \mathrm{M}$ chloroquine (Sigma, C6628), and collected supernatant from transfected cells after $48 \mathrm{~h}$. Collected supernatant containing viral solutions was ultracentrifuged yielding concentrated solution of viral particles $\left(25,000 \mathrm{rpm}\right.$ for 2 hours at $\left.4^{\circ} \mathrm{C}\right)$. Approximately $10^{7}$ 
transducing units per milliliter $(\mathrm{TU} / \mathrm{ml})$ viral solution was injected into the lateral ventricular of neonatal mouse pups (P3). After hypothermic anesthesia, viral solutions (5 $\mu \mathrm{l})$ were slowly injected using IM-9B Narishige microinjector with $2 \mu \mathrm{l} / \mathrm{min}$ speed. After recovery on the warming pad, the pups were placed back to the cage. After additional 2 hours, mice were subject to intracardiac perfusion fixation using $4 \%$ paraformaldehyde in PBS.

DAPT injection. $10 \mu \mathrm{M}$ DAPT was injected into neonatal mouse pups (P3) into a lateral ventricle (10 $\mu$ in each hemisphere). DMSO was injected into control mice. After 7 hours, mice were subject to fixation procedure using intracardiac perfusion of $4 \%$ paraformaldehyde in PBS.

Immunohistochemistry. Mice were perfused with 4\% paraformaldehyde in PBS (pH7.4) and the brains were subject to postfixation in the same fixative for $24 \mathrm{~h}$. Brains were then cryoprotected in $30 \%$ sucrose in PBS, sectioned serially $(20 \mu \mathrm{m})$ onto Superfrost plus glass slides (Fisher Scientific; Pittsburgh, PA). The brain slices were permeabilized and blocked with PBS solution containing $3 \%$ goat serum albumin and $0.3 \%$ Triton-X100, and then treated with anti-Ncadherin (1:200, Thermofisher), anti-Notch (1:200, Thermofisher), anti-PS1 (1:50), and anti-beta III tubulin (1:500, Abcam) overnight at $4^{\circ} \mathrm{C}$. The brain slices were washed three times with PBS and treated with secondary antibodies (1:1000, Thermofisher) for 30 min. Subsequently, the slices were washed with PBS, mounted and observed with a confocal microscope (Olympus, Fluoview 3000).

\section{Image Processing and Analysis}

Cadherin junction colocalization analysis. Colocalization analysis was carried out in ImageJ, using thresholding to identify AJs and then applying the JACOP plugin to quantify colocalization using Pearson coefficient, Manders' overlap coefficients, and cross-correlation analysis.

Confocal 3D z-stack image processing. Custom python code was used for automatic segmentation and junction intensity ratio analysis for Notch activation and truncation studies. Code is available at (https://github.com/kmsouthard/JunctionAnalysis) In brief, resliced z-stacks of cell-cell interfaces were thresholded to identify the AJs and membrane Notch signal. To minimize the domination of high Notch intensity, we identified the membrane expressing Notch using a minimal threshold of membrane intensity just above background. An unbiased signal analysis window along each side the junction was selected, and the Notch membrane intensity was measured for each cell by averaging along the respective windows, while junctional intensity was measured within segments determined by cadherin junctional intensity. The ratio of junctional intensity was calculated as ratio $=I_{\text {junc }} /\left(I_{\text {cell1 }}+I_{\text {cell2 }}\right)$ as deviations from the expected intensity at the junction is a function of the sum of each cell's expression level.

Intracellular mCherry nuclear translocation analysis. mCherry nuclear translocation analysis was carried out in ImageJ. GFP images were used for automated identification of cell edges and segmentation of single cells. DAPI images were used for automated identification of nucleus by implementing the Otsu thresholding method. Nuclear mCherry fluorescence data was extracted from nuclear segments by calculating the integrated fluorescence within the nucleus and subtracting a background scattering signal. In Fig. 4h, nuclear mCherry fluorescence intensities for NEXT cells treated with the macromolecular pendants were rescaled to make the intensity of $\mathrm{N}^{\mathrm{FL}}$ and NEXT to 0.002 and 1.0, respectively, which are identical to the normalized band intensities of $\mathrm{N}^{\mathrm{FL}}$ and NEXT measured by western blot.

Quantification of single-cell fluorescence. Single-cell tracking and nuclear mCherry fluorescence signal analysis of UAS-Gal4 reporter cells were performed with ImageJ, as previously described ${ }^{54,64,65}$.

Western blot quantification. Quantification of band intensities by densitometry was carried out using the Image Lab software (Bio-Rad). Band intensities of NICD in each lane were normalized by band intensities of loading control, $\beta$-actin in the corresponding lane.

\section{Estimation of protein heights}

Protein heights including extended Notch height was estimated by measuring the structural size of each domain (i.e., EGF, NRR, SNAP) in Pymol (The PyMOL Molecular Graphics System, Version 2.0 
Schrödinger, LLC.) and then creating an additive estimate based on the number of domains in the fulllength Notch construct and each Notch truncation.

\section{Molecular dynamics simulations}

All MD simulations were conducted using the GROMACS package ${ }^{96}$. Polarized MARTINI 2.2 parameters were used for the simulations ${ }^{97}$. The system was composed of 54 PPCS, 54 DPPC, 108 DPPS, 288 DIPC, $216 \mathrm{CHOL}$, and 10108 water molecules with a molar composition of lipid was CHOL:PPCS:DPPC:DIPC = $3.0: 1.5: 1.5: 4.0$ in upper layer and CHOL:DPPS:DIPC = $3.0: 3.0: 4.0$ in lower layer. To create immobilized lipids, we increased the mass of the phosphorus atom within DPPS by a factor of 1,000, keeping everything else the same in the parameter file. The pressure was set at 1.0 bar with a semi-isotropic parrinello-rahman coupling with compressibility $4.5 \times 10^{-5}$ bar $^{-1}$ and the temperature was set to $295 \mathrm{~K}$ using nose-hoover coupling. Each system was neutralized and brought to a concentration of $0.15 \mathrm{M}$ with randomly placed sodium and chloride ions. We employed the LINCS algorithm to constrain to bond lengths ${ }^{98}$. A time step of 20 fs was used with an update of the neighbor list every 10 steps, which are typical values employed in MARTINI simulations. Each simulation was run afterwards for $12 \mu \mathrm{s}$, the last $3 \mu \mathrm{s}$ of which was used for analysis. The MD simulations were analyzed using the in-built GROMACS tools. MDAnalysis libraries ${ }^{99,100}$ were used for calculating diffusion constant of lipid component and g_energy was used for calculating interlayer interaction.

\section{Statistical analysis.}

Statistical analysis was performed in GraphPad Prism 8.0 (GraphPad) or Microsoft Excel. Figure legends indicate all statistical tests used in the figure. Unless otherwise noted in the figure legends, statistical differences were determined using Student's t-test (two-tailed unpaired or paired t-test, depending on the experiment) when only two groups were compared or by ordinary one-way ANOVA followed by Tukey posthoc test when multiple groups were analyzed. The number of samples ('n') used for each experimental analysis is indicated in the figure legends. Sample sizes of sufficient power were chosen on the basis of general standards accepted by the field. In all cases, statistical significance was assumed for probability $(\mathrm{P})<0.05$. ${ }^{*} \mathrm{P}<0.05$; ${ }^{* *} \mathrm{P}<0.01$; ${ }^{* *} \mathrm{P}<0.001$; ${ }^{* * *} \mathrm{P}<0.0001$; ns indicates when no significant difference was detected.

87. Kim, J. et al. Single-cell mechanogenetics using monovalent magnetoplasmonic nanoparticles. Nature Protocols 2017 12:9 12, 1871-1889 (2017).

88. Moss, M. L. \& Rasmussen, F. H. Fluorescent substrates for the proteinases ADAM17, ADAM10, ADAM8, and ADAM12 useful for high-throughput inhibitor screening. Analytical Biochemistry 366, 144148 (2007).

89. Lee, H. J. et al. En bloc and segmental deletions of human XIST reveal X chromosome inactivation-involving RNA elements. Nucleic Acids Research 47, 3875-3887 (2019).

90. Pulido-Quetglas, C. et al. Scalable Design of Paired CRISPR Guide RNAs for Genomic Deletion. PLOS Computational Biology 13, e1005341 (2017).

91. Kim, H. K. et al. SpCas9 activity prediction by DeepSpCas9, a deep learning-based model with high generalization performance. Science Advances 5, eaax9249 (2019).

92. Ramakrishna, S. et al. Gene disruption by cell-penetrating peptide-mediated delivery of Cas 9 protein and guide RNA. Genome Research 24, 1020-1027 (2014).

93. Ramakrishna, S. et al. Surrogate reporter-based enrichment of cells containing RNA-guided Cas9 nuclease-induced mutations. Nature Communications 5, 3378 (2014). 
94. Gopalappa, R., Suresh, B., Ramakrishna, S. \& Kim, H. (Henry). Paired D10A Cas9 nickases are sometimes more efficient than individual nucleases for gene disruption. Nucleic Acids Research 46, e71e71 (2018).

95. Guschin, D. Y. et al. A Rapid and General Assay for Monitoring Endogenous Gene Modification. Methods in Molecular Biology 649, 247-256 (2010).

96. van der Spoel, D. et al. GROMACS: Fast, flexible, and free. Journal of Computational Chemistry 26, 1701-1718 (2005).

97. de Jong, D. H. et al. Improved Parameters for the Martini Coarse-Grained Protein Force Field. Journal of Chemical Theory and Computation 9, 687-697 (2013).

98. Hess, B., Bekker, H., Berendsen, H. J. C. \& Fraaije, J. G. E. M. LINCS: A linear constraint solver for molecular simulations. Journal of Computational Chemistry 18, 1463-1472 (1997).

99. Michaud-Agrawal, N., Denning, E. J., Woolf, T. B. \& Beckstein, O. MDAnalysis: A toolkit for the analysis of molecular dynamics simulations. Journal of Computational Chemistry 32, 2319-2327 (2011).

100. Chavent, M. et al. Methodologies for the analysis of instantaneous lipid diffusion in md simulations of large membrane systems. Faraday Discussions 169, 455-475 (2014).

\section{Acknowledgement}

The authors thank Drs. S. Blacklow (Harvard U.), C. Miller (King's College London), and K. Shimamura (Kumamoto U.) for the kind gifts of Notch, APP, and DN-cadherin plasmids, respectively. We also thank Drs. A. Balmain, M. Moasser, and E. Collison (UCSF) for sharing cell lines. Dr. Daniel Fletcher (UC Berkeley), Mr. Ari Joffe (UC Berkeley), and Dr. Duaa Al-Rawi (Stanford U.) provided insightful discussion. For reagents, technical support, and discussions we thank the Kim, Cheon, Gartner, and Jun laboratories, as well as the Nikon Imaging Center and Wynton at UCSF. M.K. was supported by a Life Science Research Foundation fellowship as the Shurl and Kay Curci Foundation fellow, and by Burroughs Wellcome Travel Fund. This work was supported by $2020 R 1 A 2 C 4002533$ (D.S.) and NRF2017R1A2B3004198 (H.K.), HI17C0676 from Korean Ministry of Health and Welfare (H.K.), 5R01AG008200 from National Institute on Aging (NIA) and the National Institute of Health (NIH) (N.K.R.), IBS-R026-D1 from IBS (M.K., H.K., and J.C.), NRF-2019R1A2C1085712 (Y.H.K.), the UCSF Center for Cellular Construction (an NSF Science and Technology Center, no. DBI-1548297) (Z.J.G.), U01CA244109 from the National Cancer Institute (Z.J.G), 1R01GM112081, 1R01GM126542-01, and R35GM134948 from the National Institute of General Medical Science (NIGMS) and the NIH (Y.J.), 1R21AG072232-01 from the National Institute on Aging (NIA) and the NIH (M.L.K. and Y.J.) and the UCSF Program for Breakthrough Biomedical Research (PBBR) funded in part by the Sandler Foundation (M.L.K and Y.J.). Z.J.G. is a Chan Zuckerberg BioHub Investigator.

\section{Author Contributions}

M.K., K.M.S., Z.J.G., and Y.J. conceived the ideas and designed research; M.K. and K.M.S. constructed plasmids, generated cell lines, and performed confocal microscopy. M.K. performed mechanogenetics, truncation study, spatial mutation, immunoblot analysis, reporter cell assay, and APP experiment. K.M.S. performed Notch exclusion and activation experiments, designed truncation study, wrote custom python image analysis scripts. W.R.K. performed animal experiment, flotillin staining, and mechanogenetic experiment. N.K. performed coarse-grained MD simulation. R.G. generated cadherin-KO cells. M.A. synthesized magnetic nanoparticles. H.L. helped confocal imaging/western blot analysis, respectively. M.K.K. performed Elisa analysis of $A \beta$ secretion from $C D H$ KO cells. S.H.C. performed western blot analysis. J.F. and D.S. performed initial proof-of-concept experiments. A.G. and N.K.R. provided antiPS1 antibodies. M.L.K. helped VE-cad experiment, H.K., Y.H.K., and J.C. oversaw CRISPR-Cas9 KO experiment, MD simulation, and magnetic nanoparticle synthesis, respectively. Z.J.G. oversaw and 
supervised all spatial mapping and Notch truncation experiments. Y.J. oversaw and supervised all aspects of the study. M.K. and K.M.S. analyzed data. M.K., K.M.S., Z.J.G., and Y.J. wrote the manuscript.

\section{Ethics Declaration}

The authors declare no competing interest.

\section{Supplementary Materials}

Supplementary Notes

Supplementary Table 1

Extended Data Figures 1-7

Supplementary Videos S1, S2 


\section{Supplementary Files}

This is a list of supplementary files associated with this preprint. Click to download.

- SupplementaryInformationfinal2.pdf

- Movie1.avi

- Movie2.avi 